\title{
Little strings and T-duality
}

\author{
Jungmin Kim, ${ }^{a}$ Seok $\operatorname{Kim}^{a}$ and Kimyeong $\operatorname{Lee}^{b}$ \\ ${ }^{a}$ Department of Physics and Astronomy $\&$ Center for Theoretical Physics, \\ Seoul National University, \\ 1 Gwanak-ro, Seoul 151-747, Korea \\ ${ }^{b}$ School of Physics, Korea Institute for Advanced Study, \\ 85 Hoegiro, Seoul, Korea
}

E-mail: kjmint82@gmail.com, skim@phya.snu.ac.kr, klee@kias.re.kr

Abstract: We study the $2 \mathrm{~d} \mathcal{N}=4$ gauge theory descriptions of little strings on type II NS5-branes. The IIB strings on $N$ NS5-branes are described by the $\mathcal{N}=(4,4)$ gauge theories, whose Higgs branch CFTs on $\mathrm{U}(N)$ instanton moduli spaces are relevant. The IIA strings are described by $\mathcal{N}=(4,4) \hat{A}_{N-1}$ quiver theories, whose Coulomb branch CFTs are relevant. We study new $\mathcal{N}=(0,4)$ quiver gauge theories for the IIA strings, which make it easier to study some infrared observables. In particular, we show that the supersymmetric partition functions of the IIA/IIB strings on Omega-deformed $\mathbb{R}^{4} \times T^{2}$ precisely map to each other by T-duality.

KEYwords: Supersymmetric gauge theory, Supersymmetry and Duality, Brane Dynamics in Gauge Theories, String Duality

ARXIV EPRINT: 1503.07277 


\section{Contents}

1 Introduction 1

2 IIB little strings $\quad 3$

2.1 A brief review 3

2.2 The elliptic genus of IIB little strings 5

3 IIA little strings $\quad 7$

$\begin{array}{ll}3.1 \mathcal{N}=(0,4) \text { gauge theory descriptions } & 10\end{array}$

$\begin{array}{ll}3.2 & \text { The elliptic genus of IIA little strings } \\ \end{array}$

4 T-duality of protected little string spectra $\quad 16$

$\begin{array}{lll}4.1 & \text { One NS5-brane } & 19\end{array}$

4.2 Two NS5-branes 22

4.3 Three NS5-branes 26

$5 \quad \operatorname{SL}(2, Z)$ transformations of the elliptic genus $\quad 30$

6 Concluding remarks $\quad 31$

A $\mathcal{N}=(4,4)$ gauge theory of IIB strings 32

\section{Introduction}

Little string theories with 16 supercharges [1-5] are obtained by decoupling limits of the type II strings near N NS5-branes. These are non-local theories without gravity. Depending on whether we start from type IIA or IIB NS5-branes, the system has $(2,0)$ or $(1,1)$ super-Poincare symmetry, respectively. Since NS5-branes are one of the most difficult nonperturbative objects to study in string theory, it would be very desirable to have better understanding on these strings. Also, the type IIA little string theory has interesting low energy limit given by interacting $(2,0)$ superconformal field theories. Little strings have similarities with critical strings, and also differences. The fact that these models do not contain gravity is the main difference, with far-reaching implications. However, being nonlocal theories, such systems inherit from the type II strings various stringy properties, such as the T-duality. So after circle compactification, the two little string theories are supposed to be T-dual to each other.

Unlike critical strings, noncritical little strings are difficult to study. Some approaches to study them are: holographic approach [3, 4], discrete lightcone quantization $[1,2,6,7]$, the double scaling limit [8,9], studies on the scattering amplitudes and higher derivative terms in the effective action $[10,11]$. In particular, the DLCQ approach considers the little 
string theory compactified on a small circle, in which one studies a sector with definite momentum which is decoupled from the rest. Via T-duality, the DLCQ description can be obtained by a large radius compactification of the T-dual strings with definite winding number.

In this paper, we study the QFTs living on the little strings macroscopically extended on $\mathbb{R}^{1,1}$. They describe $2 \mathrm{~d}$ decoupled degrees of freedom living on these strings at low energy. Such theories are studied in detail in the literature [6, 7]. One starts from $2 \mathrm{~d} \mathcal{N}=(4,4)$ gauge theories, which flow to interacting CFTs and describe these strings. Compactifying these strings on large circles, the ground state energy is proportional to the radius times the winding quantum number, much larger than the energy scale of the circle momenta. So we can consider a low energy decoupled sector with fixed winding quantum numbers. They also have direct relevance to the study of DLCQ little strings obtained after T-duality, in which the momentum is fixed. In this paper, we make a modest contribution to construct and study such UV gauge theories for the little strings, on the IIA side starting from $\mathcal{N}=(0,4)$ gauge theories. The system is proposed to flow to a CFT with enhanced $(4,4)$ SUSY. Compared to the $(4,4)$ gauge theories discussed in [7], the new description has an advantage of manifestly having certain IR symmetries in UV, which is very crucial for computing some protected IR observables such as the elliptic genus. The $(0,4)$ UV QFTs are similar to those for the self-dual strings of the $(2,0)$ superconformal field theory, called 'M-strings' [15].

With T-duality, the spectrum of the circle compactified theories would be the same for IIA and IIB little strings. We would like to probe this T-duality with the above gauge theory descriptions for macroscopic strings. In general, these descriptions are valid only when the compactification radii are large. As the T-duality exchanges the IIA and IIB radii as $R_{A}=\frac{\alpha^{\prime}}{R_{B}}$, the two gauge theory descriptions will never be simultaneously reliable. However, one naturally expects that the protected BPS spectrum would be reliable all the way to small radii.

In this paper, we study the T-duality of little strings in the BPS sector, from the UV gauge theory descriptions. In particular, being able to compute the elliptic genera of the $2 \mathrm{~d}$ gauge theories on both IIA and IIB sides, we can directly compare their BPS spectra. More precisely, we shall compare the full BPS partition functions of the IIA/IIB little strings on Omega-deformed $\mathbb{R}^{4} \times T^{2}$ (or supersymmetric indices of circle compactifieid theories with nonzero chemical potentials), which are closely related to the elliptic genera of our gauge theories. We find, in fugacity expansions to highly nontrivial orders, that the two partition functions precisely map to each other via T-duality. ${ }^{1}$ Apart from confirming the naturally expected T-duality, our finding is establishing a very nontrivial identity between the elliptic genera computed from the type IIA and IIB sides, so that alternative expressions can be used to extract various properties which would have been very difficult to see from the other viewpoints. For instance, we explain in section 5 how one can easily understand the $\mathrm{SL}(2, \mathbb{Z}) \times \mathrm{SL}(2, \mathbb{Z})$ transformation properties of the partition functions, for the complex structure and Kahler parameters of the torus, by using our T-dual expressions.

\footnotetext{
${ }^{1}$ In order to better define our spectral problem, without continua coming from the 'throat' regions [6, 7 , 12], we turn on the Fayet-Iliopoulos (FI) term and the theta angle of the gauge theories on the worldsheet. Also, to avoid having infrared problems with tensionless fractional strings or W-bosons, we separate the $N$ NS5-branes and study the massive spectra.
} 
The rest of this paper is organized as follows. In sections 2 and 3, we explain the $2 \mathrm{~d}$ gauge theory descriptions of the IIB and IIA little strings, respectively, and study their elliptic genera. In section 4, we study the T-duality of the two Omega-deformed partition functions, as well as extended duality/triality properties. In section 5 , we study the $\mathrm{SL}(2, \mathbb{Z})$ transformation properties of the elliptic genus in various fugacities. Section 6 concludes with brief discussions.

\section{IIB little strings}

\subsection{A brief review}

We first consider the type IIB little strings, which are the type IIB fundamental strings bound to the NS5-branes. At low energy, the world-volume description of IIB NS5-branes is given by $6 \mathrm{~d}$ maximally supersymmetric Yang-Mills theory, with $(1,1)$ supersymmetry and $\mathrm{U}(N)$ gauge group. The fields consist of the gauge field $A_{\mu=0, \cdots, 5}, 4$ scalar fields $\phi^{I=1, \cdots, 4}$, and fermions. These degrees of freedom are provided by the D-strings ending on the NS5branes. The bosonic symmetry of the theory is $\mathrm{SO}(1,5) \times \mathrm{SO}(4)_{R} \cdot \mathrm{SO}(1,5)$ is the Lorentz symmetry on the NS5-branes, and $\mathrm{SO}(4)_{R}$ is the symmetry on their transverse directions, which rotates $\phi^{I}$. The Yang-Mills coupling constant is given by

$$
g_{\mathrm{YM}}^{2}=\frac{1}{T_{\mathrm{NS} 5}\left(2 \pi \alpha^{\prime}\right)^{2} g_{s}^{2}}=(2 \pi)^{3} \alpha^{\prime}
$$

Fundamental strings form threshold bounds with the NS5-branes. They are identified as the instanton strings in the 6d SYM. The instanton string tension is given by

$$
\frac{4 \pi^{2}}{g_{\mathrm{YM}}^{2}}=\frac{1}{2 \pi \alpha^{\prime}}=T_{\mathrm{F} 1},
$$

agreeing with the tension of the fundamental string. The coupling constant is independent of the $10 \mathrm{~d}$ string coupling constant, $g_{s}$. So one can take the little string theory limit, in which we take $g_{s} \rightarrow 0$ with fixed $\alpha^{\prime}$. All the gravitational degrees of freedom are decoupled.

We shall consider $k$ macroscopically extended little strings, extended along $\mathbb{R}^{1,1}$ part of $\mathbb{R}^{5,1}$. We are interested in the dynamics of the degrees of freedom supported on these macroscopic strings, decoupled from the rest of the $6 \mathrm{~d}$ degrees of freedom at low energy. The system of $k \mathrm{~F} 1$ and $N$ NS5-branes admit a UV gauge theory description given by a $\mathrm{U}(k)$ gauge theory with $\mathcal{N}=(4,4)$ supersymmetry. The field theory is identical to that living on the D1-D5 system via S-duality, and has been studied extensively in the literature, e.g. [6, $7,12]$. This $2 \mathrm{~d}$ theory at low energy can also be regarded as the worldsheeet description of the instanton strings of the 6d SYM theory. The gauge theory has the $\mathrm{U}(k) \mathcal{N}=$ $(4,4)$ vector multiplet, an adjoint hypermultiplet, and $N$ fundamental hypermulitiplets which rotate under $\mathrm{U}(N)$ global symmetry. These fields are shown in table 1, and more details about this theory is explained in appendix A. For later convenience, we also show the supermultiplet structure with respect to the right-chiral $(0,4)$ SUSY. The bosonic symmetry preserved by the strings is $\mathrm{SO}(1,1) \times \mathrm{SO}(4) \subset \mathrm{SO}(1,5)$ times $\mathrm{SO}(4)_{R}$, where the 


\begin{tabular}{|c|c|c|c|c|}
\hline $\mathcal{N}=(4,4)$ & $\mathcal{N}=(0,4)$ & Fields & $\mathrm{U}(k)$ & $\mathrm{U}(N)$ \\
\hline \multirow{2}{*}{ vector } & vector & $A_{\mu}, \bar{\lambda}_{+}^{A \dot{\alpha}}$ & adj & 1 \\
\cline { 2 - 5 } & twisted hyper & $\varphi_{a A}, \bar{\lambda}_{a-}^{\dot{\alpha}}$ & adj & 1 \\
\hline \multirow{2}{*}{ hyper } & hyper & $a_{\alpha \dot{\beta}}, \lambda_{\alpha-}^{A}$ & adj & 1 \\
\cline { 2 - 5 } & Fermi & $\lambda_{a \beta+}$ & adj & 1 \\
\hline \multirow{2}{*}{ hyper } & hyper & $q_{\dot{\alpha}}, \psi_{-}^{A}$ & $\overline{\mathbf{k}}$ & $\mathbf{N}$ \\
\cline { 2 - 5 } & Fermi & $\psi_{a-}$ & $\overline{\mathbf{k}}$ & $\mathbf{N}$ \\
\hline
\end{tabular}

Table 1. $\mathcal{N}=(4,4)$ supermultiplets for $k$ IIB strings.

latter is inherited from the R-symmetry of the $6 \mathrm{~d}$ theory. For $\mathrm{SO}(4) \sim \mathrm{SU}(2)_{L 1} \times \mathrm{SU}(2)_{R 1}$ and $\mathrm{SO}(4)_{R} \sim \mathrm{SU}(2)_{L 2} \times \mathrm{SU}(2)_{R 2}$, we introduce the following doublet indices,

$$
\mathrm{SU}(2)_{L 1} \rightarrow \alpha, \mathrm{SU}(2)_{R 1} \rightarrow \dot{\alpha}, \mathrm{SU}(2)_{L 2} \rightarrow a, \mathrm{SU}(2)_{R 2} \rightarrow A
$$

The fields in table 1 and appendix A are given with this convention. The $6 \mathrm{~d}(1,1)$ supercharges can be written as $Q_{a \alpha+}, Q_{\alpha-}^{A}, Q_{a+}^{\dot{\alpha}}, Q_{-}^{A \dot{\alpha}}$, where \pm denote $6 \mathrm{~d}$ chirality. These supercharges satisfy the reality conditions given by

$$
Q_{a \alpha+}=-\epsilon_{\alpha \beta} \epsilon_{a b}\left(Q_{b \beta+}\right)^{\dagger}, Q_{\alpha-}^{A}=\epsilon_{\alpha \beta} \epsilon^{A B}\left(Q_{\beta-}^{B}\right)^{\dagger}, Q_{a+}^{\dot{\alpha}}=-\epsilon^{\dot{\alpha} \dot{\beta}} \epsilon_{a b}\left(Q_{b+}^{\dot{\beta}}\right)^{\dagger}, Q_{-}^{A \dot{\alpha}}=\epsilon^{\dot{\alpha} \dot{\beta}} \epsilon^{A B}\left(Q_{-}^{B \dot{\beta}}\right)^{\dagger} .
$$

The strings extended on $\mathbb{R}^{1,1}$ preserve $Q_{a+}^{\dot{\alpha}}$ and $Q_{-}^{A \dot{\alpha}}$, forming $2 \mathrm{~d} \mathcal{N}=(4,4)$ supersymmetry. The \pm subscripts on $2 \mathrm{~d}$ fermions denote left/right chiralities, respectively. In table 1 , the fields $a_{\alpha \dot{\beta}}$ and $q_{\dot{\alpha}}$ form the so-called ADHM data of $k$ multi-instantons of $\mathrm{U}(N)$ gauge theory. This is because the IR limit of this gauge theory will be describing the $6 \mathrm{~d}$ instanton strings, as we shall explain in more detail now.

The infrared dynamics of this $(4,4)$ theory has been studied in [6]. Its low energy dynamics is described by two decoupled $(4,4)$ conformal field theories. One is the conformal field theory on the Higgs branch described by a nonlinear sigma model on the Higgs branch target space, given by $k$ instanton moduli space. Another is the conformal field theory on the Coulomb branch. For studying the type IIB little strings, the Higgs branch CFT is of relevance. The Coulomb branch degrees of freedom $\varphi_{a A}$ represent the motion of the strings moving away from the 5-branes.

There is a peculiar singularity in the region near $q_{\dot{\alpha}}=0, a_{\alpha \dot{\beta}}=0$, where the Higgs branch classically meets the Coulomb branch [6, 7, 12]. Quantum mechanically, this region forms a 'throat,' which is responsible for a continuum in the CFT spectrum. The CFT can be deformed by turning on the $\mathrm{SU}(2)_{R 1}$ triplet of Fayet-Iliopoulos term $\zeta^{I}(I=1,2,3)$ and the theta angle $\theta$, so that such continuum disappears $[6,7,12]$. In particular, the Higgs branch moduli space becomes regular, and the Coulomb branch is no longer connected to the Higgs branch even classically. We shall discuss the little string spectrum with nonzero FI term, by studying the elliptic genus $[16]$ of the $2 \mathrm{~d}(4,4)$ gauge theory. (The continuum will be completely lifted, not only by the FI-term but also by the Coulomb VEV of the $6 \mathrm{~d}$ SYM which removes the infrared continuum.) In particular, since the FI parameter gives 
nonzero energy to the Coulomb branch [17], the elliptic genus will acquire contribution only from the Higgs branch CFT for the IIB little strings, and not from the Coulomb branch CFT that we are not interested in.

\subsection{The elliptic genus of IIB little strings}

In this subsection we shall define and explain the elliptic genus of the gauge theory compactified on circle, counting $\frac{1}{4}$-BPS states in the Coulomb phase of the $6 \mathrm{~d}$ theory, which shall be further studied in sections 4 and 5 . This is a supersymmetric partition function of our $(4,4)$ theory on a torus with complex structure $\tau$. We choose a supercharge $Q=Q^{A=1, \dot{\alpha}=\dot{2}}$ and its conjugate $Q^{\dagger}$ and define its index, with $q \equiv e^{2 \pi i \tau}$,

$$
\begin{aligned}
Z_{\text {inst }}^{\mathrm{IIB}}\left(\alpha_{i}, \epsilon_{ \pm}, m ; q, w\right) & =\operatorname{Tr}\left[(-1)^{F} w^{k} q^{H_{L}} \bar{q}^{H_{R}} e^{2 \pi i \alpha_{i} \Pi_{i}} e^{2 \pi i \epsilon_{-}\left(2 J_{1 L}\right)} e^{2 \pi i m\left(2 J_{2 L}\right)} e^{2 \pi i \epsilon_{+}\left(2 J_{1 R}+2 J_{2 R}\right)}\right] \\
& =\sum_{k=0}^{\infty} w^{k} Z_{k}\left(\alpha_{i}, \epsilon_{ \pm}, m ; q\right)
\end{aligned}
$$

with $Z_{0} \equiv 1$. The elliptic genus is using only the $(0,2)$ subset of $(4,4)$ supercharges. $P$ is the momentum on the string compactified on the circle, and $H$ is the energy, in the unit of inverse-radius $R_{B}^{-1}$ of the circle. $2 H_{L}=H+P$ and $2 H_{R}=H-P$ are defined as the leftmoving and rightmoving momentum, respectively. $J_{L 1,2}$ and $J_{R 1,2}$ are the Cartans of $\mathrm{SU}(2)_{L 1,2}$ and $\mathrm{SU}(2)_{R 1,2}$. Since $\left\{Q, Q^{\dagger}\right\}=2 H_{R}$ and $Q$ commutes with all the other factors in the trace, the index counts only the BPS states annihilated by $Q$ and $Q^{\dagger}$, and it is independent of $\bar{q} . \Pi_{i}$ 's are the Cartans of $\mathrm{U}(N) . \quad \alpha_{i}$ 's are the chemical potentials for electric charges, interpreted as the background gauge field $A_{5}=\operatorname{diag}\left(\left\{\alpha_{i}\right\}\right)$ along the spatial circle, breaking $\mathrm{U}(N)$ to $\mathrm{U}(1)^{N}$. We also introduce the fugacity variable, $w$, counting the winding number $k$ of the little strings. For a given $\mathrm{U}(k)$ gauge theory, we fix $k$ and compute $Z_{k}$. The above index is the grand partition function. We use the subscript 'inst' standing for 'instantons' in the 6d SYM interpretation.

$Z_{k}$ can be computed from the $\mathcal{N}=(4,4)$ theory explained in section 2.1 and appendix $\mathrm{A}$, as follows. The gauge theory elliptic genus can be computed by performing a suitable contour integral of [18-20], with the integral measure given by the contributions from the fields listed in table 1 . The integral representation for $Z_{k}$ is given by [21]

$$
\begin{aligned}
Z_{k} & =\frac{1}{k !} \oint\left[\prod_{I=1}^{k} \frac{2 \pi \eta^{2} d u_{I}}{2 \pi i}\right] Z_{\mathrm{vec}}\left(u, \alpha, \epsilon_{ \pm}\right) Z_{\mathrm{hyper}}\left(u, \alpha, \epsilon_{ \pm}, m\right) \\
Z_{\mathrm{vec}} & =\frac{\prod_{I \neq J} \theta_{1}\left(u_{I J}\right) \prod_{I, J=1}^{k} \theta_{1}\left(u_{I J}+2 \epsilon_{+}\right)}{\prod_{I=1}^{k} \prod_{i=1}^{N} \theta_{1}\left(\epsilon_{+} \pm\left(u_{I}-\alpha_{i}\right)\right) \prod_{I, J=1}^{k} \theta_{1}\left(\epsilon_{1,2}+u_{I J}\right)} \\
Z_{\mathrm{hyper}} & =\frac{\prod_{I=1}^{k} \prod_{i=1}^{N} \theta_{1}\left(m \pm\left(u_{I}-\alpha_{i}\right)\right) \prod_{I, J=1}^{k} \theta_{1}\left( \pm m-\epsilon_{-}+u_{I J}\right)}{\prod_{I, J=1}^{k} \theta_{1}\left( \pm m-\epsilon_{+}+u_{I J}\right)} .
\end{aligned}
$$

$Z_{\text {vec }}$ comes from the zero modes of $6 \mathrm{~d} \mathcal{N}=1$ vector multiplet in the $k$ instanton backgrounds, which are the first, third, fifth lines of table $1 . Z_{\text {hyper }}$ comes from the zero modes of $6 \mathrm{~d}$ adjoint hypermultiplet (which makes the $6 \mathrm{~d} \mathcal{N}=2$ vector multiplet) in the instanton 
backgrounds, which are the second, fourth, sixth lines. The contour integral can be done by following precisely the same steps as explained in [21], which did the Witten index calculus for the $1 \mathrm{~d}$ reduction of our $(4,4)$ theory, to count instantons in the $5 \mathrm{~d} \mathcal{N}=1^{*}$ theory. One can repeat the analysis in the elliptic version. After this analysis, $Z_{k}$ is given by a sum of residues which are labeled by the $N$-colored Young diagrams, $Y=\left\{Y_{1}, Y_{2}, \cdots, Y_{N}\right\}$. The sum of the numbers of the boxes $\sum_{i=1}^{N}\left|Y_{i}\right|$ is $k$. The elliptic genus is given by

$$
Z_{k}\left(\alpha_{i}, \epsilon_{ \pm}, m ; q\right)=\sum_{Y: \sum_{i}\left|Y_{i}\right|=k} \prod_{i, j=1}^{N} \prod_{s \in Y_{i}} \frac{\theta_{1}\left(q ; E_{i j}+m-\epsilon_{-}\right) \theta_{1}\left(q ; E_{i j}-m-\epsilon_{-}\right)}{\theta_{1}\left(q ; E_{i j}-\epsilon_{1}\right) \theta_{1}\left(q ; E_{i j}+\epsilon_{2}\right)}
$$

where

$$
E_{i j}=\alpha_{i}-\alpha_{j}-\epsilon_{1} h_{i}(s)+\epsilon_{2} v_{j}(s) .
$$

' $s$ ' denotes a box in the Young diagram $Y_{i} . h_{i}(s)$ is the distance from the box ' $s$ ' to the edge on the right side of $Y_{i}$ that one reaches by moving horizontally. $v_{j}(s)$ is the distance from ' $s$ ' to the edge on the bottom side of $Y_{j}$ that one reaches by moving vertically. See e.g. [22] for more details and illustrations. This Young diagram expression first appeared in the instanton calculus of the $4 \mathrm{~d}$ and 5d SYM [23, 24], and our result (2.7) is simply an elliptic uplift of their results. Note also that the expression (2.7) at $N=1$ appeared in [25].

For later use, it would be helpful to note the key steps towards the derivation of (2.7). Firstly, the contour integral in (2.6) is a sum over the so-called Jeffrey-Kirwan residues (JK-Res) [20]. The poles with nonzero JK-Res for (2.6) has been studied in [21]. It was

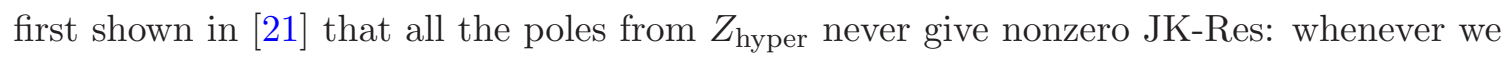
pick a pole from the denominator term $\theta_{1}\left( \pm m-\epsilon_{+}+u_{I J}\right)$ according to the rules of [20,21], a term of the form $\theta_{1}\left( \pm m-\epsilon_{-}+u_{I J}\right)$ in the numerator always vanishes. So we can completely restrict our discussions to the poles from $Z_{\mathrm{vec}}$. Then the poles from $Z_{\text {vec }}$ with nonzero JK-Res are completely classified, and is shown to be labeled by the $N$ Young diagrams that we explained above. Then, we insert the pole values of $u_{I}$, labeled by $Y$, to extract out the JK-Res. Within $Z_{\text {vec }}$ and $Z_{\text {hyper }}$, there are vast cancelations between the $\theta_{1}$ functions in the numerator and denominator $[23,24]$. From $Z_{\mathrm{vec}}$, only bosonic terms remain, which explain the denominator of (2.7). From $Z_{\text {hyper }}$, only fermionic terms remain, explaining the numerator of (2.7).

It is also useful to consider the full index of the type IIB little string theory, compactified on a circle with large radius $R_{B} \gg\left(\alpha^{\prime}\right)^{\frac{1}{2}}$. The index is defined in the same way as (2.5), where the trace is taken over the whole BPS Hilbert space of the $6 \mathrm{~d}$ theory in the Coulomb phase. This is a BPS partition function on $\mathbb{R}^{4} \times T^{2}$. Apart from (2.5), one finds extra contribution from the 6d perturbative SYM states, decoupled from the winding strings at low energy. The full index thus factorizes as

$$
Z_{\mathrm{IIB}}\left(\alpha_{i}, \epsilon_{ \pm}, m ; q, w\right)=Z_{\mathrm{pert}}^{\mathrm{IIB}}\left(\alpha_{i}, \epsilon_{ \pm}, m ; q\right) Z_{\mathrm{inst}}^{\mathrm{IIB}}\left(\alpha_{i}, \epsilon_{ \pm}, m ; q, w\right) .
$$

The $6 \mathrm{~d}$ perturbative index, $Z_{\text {pert }}^{\mathrm{IIB}}$, counts the modes which only carry momenta along the circle. This can be computed as follows. (See [22] for a very similar analysis of the perturbative partition function of 5d maximal SYM.) In the Coulomb phase, both 
momentum along the circle and the electric charge preserve supercharges $Q_{a \alpha+}$ and $Q_{-}^{A \dot{\alpha}}$, and breaks $Q_{a+}^{\dot{\alpha}}$ and $Q_{\alpha-}^{A}$. These are the $\frac{1}{2}$-BPS condition for the W-bosons. Without losing generality, let us order the Coulomb VEVs $\sim A_{5}=\operatorname{diag}\left(\alpha_{i}\right)$ by $0<\alpha_{1}<\alpha_{2}<\cdots<\alpha_{N}<1$ on the circle. Then the BPS W-bosons preserving the above half supercharges will carry the factor $e^{2 \pi i\left(\alpha_{i}-\alpha_{j}\right)}$ with $i>j$ and the momentum is zero $P=0$. If $P \neq 0$, only the states with $P>0$ will be BPS. The states with $P<0$ are anti-BPS and will not be captured by our index. If we take $q \ll 1$, electric charge factor $e^{2 \pi i\left(\alpha_{i}-\alpha_{j}\right)}$ with any $i, j$ can be assumed, since the central charge is still kept positive by $P>0$.

Now we compute the single-particle index of the perturbative sector. The Goldstino zero modes coming from the broken SUSY generators contribute to the single particle index with the following factor,

$$
2^{4} \sinh \frac{2 \pi i\left(m+\epsilon_{+}\right)}{2} \sinh \frac{2 \pi i\left(m-\epsilon_{+}\right)}{2} \sinh \frac{2 \pi i \epsilon_{1}}{2} \sinh \frac{2 \pi i \epsilon_{2}}{2},
$$

and the bosonic zero modes on $\mathbb{R}^{4}$ provides the factor

$$
\frac{1}{2^{4} \sinh ^{2} \frac{2 \pi i \epsilon_{1}}{2} \sinh ^{2} \frac{2 \pi i \epsilon_{2}}{2}}
$$

where $\epsilon_{ \pm} \equiv \frac{\epsilon_{1} \pm \epsilon_{2}}{2}$. Therefore, the single particle index of the perturbative particles always contains the following overall factor coming from the spacetime 0-modes [22],

$$
I_{+}\left(\epsilon_{ \pm}, m\right)=\frac{\sinh \frac{2 \pi i\left(m+\epsilon_{+}\right)}{2} \sinh \frac{2 \pi i\left(m-\epsilon_{+}\right)}{2}}{\sinh \frac{2 \pi i \epsilon_{1}}{2} \sinh \frac{2 \pi i \epsilon_{2}}{2}} .
$$

Multiplying the fugacity factors for the $\mathrm{U}(N)$ electric charges and the momentum, the single particle index of the $6 \mathrm{~d}$ perturbative particles is given by

$$
\begin{aligned}
z_{\mathrm{sp}} & =N I_{+}\left(\epsilon_{ \pm}, m\right) \cdot \sum_{n=1}^{\infty} q^{n}+I_{+}\left(\epsilon_{ \pm}, m\right) \cdot\left(\sum_{i>j}^{N} e^{2 \pi i\left(\alpha_{i}-\alpha_{j}\right)}+\sum_{i \neq j}^{N} \sum_{n=1}^{\infty} e^{2 \pi i\left(\alpha_{i}-\alpha_{j}\right)} q^{n}\right) \\
& =I_{+} \sum_{i>j}^{N} e^{2 \pi i\left(\alpha_{i}-\alpha_{j}\right)}+I_{+}\left(N+\sum_{i \neq j}^{N} e^{2 \pi i\left(\alpha_{i}-\alpha_{j}\right)}\right) \frac{q}{1-q} .
\end{aligned}
$$

The first term on the first line comes from the states with $P=n>0$ and $\Pi_{i}=0$, second term from W-bosons at $P=0$, and the last term from both electric charges and $P=n>0$. From this, $Z_{\text {pert }}^{\mathrm{IIB}}$ is given by

$$
Z_{\mathrm{pert}}^{\mathrm{IIB}}\left(\alpha_{i}, \epsilon_{ \pm}, m ; q\right)=P E\left[z_{\mathrm{sp}}\left(\alpha_{i}, \epsilon_{ \pm}, m ; q\right)\right]=\exp \left[\sum_{p=1}^{\infty} \frac{1}{p} z_{\mathrm{sp}}\left(p \alpha_{i}, p \epsilon_{ \pm}, p m ; q^{p}\right)\right] .
$$

\section{IIA little strings}

Type IIA NS5-branes realize $6 \mathrm{~d}$ IIA little string theory, with $\mathcal{N}=(2,0)$ supersymmetry. The light degrees of freedom are made of a self-dual tensor field $B_{\mu \nu}$, and 5 scalars, $\phi^{I=1,2,3,4}$ 


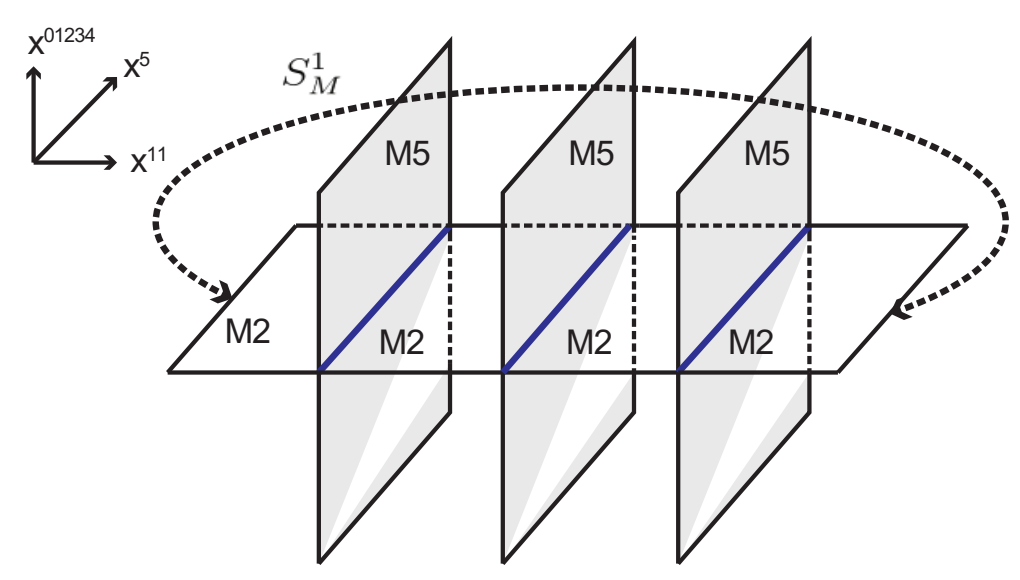

Figure 1. M-theory brane uplift of the IIA little strings.

\begin{tabular}{|c|c|c|c|c|c|c|c|c|c|c|c|}
\hline & $x^{0}$ & $x^{1}$ & $x^{2}$ & $x^{3}$ & $x^{4}$ & $x^{5}$ & $x^{6}$ & $x^{7}$ & $x^{8}$ & $x^{9}$ & $x^{11}\left(S_{\mathrm{M}}^{1}\right)$ \\
\hline$N \mathrm{M} 5$ & $\times$ & $\times$ & $\times$ & $\times$ & $\times$ & $\times$ & & & & & $\alpha_{i}$ \\
\hline$n_{i} \mathrm{M} 2$ & $\times$ & & & & & $\times$ & & & & & $\left(\alpha_{i}, \alpha_{i+1}\right)$ \\
\hline
\end{tabular}

Table 2. M-theory brane uplift of IIA little strings.

and $\phi$, and fermions. $\phi^{I=1,2,3,4}$ parametrize the transverse $\mathbb{R}^{4}$ of type IIA string theory, and $\phi$ is a compact scalar parametrizing the position of the 5-branes along the M-theory circle. The little strings are type IIA fundamental strings bound to the NS5-branes. In M-theory, type IIA fundamental strings uplift to M2-branes wrapping the M-theory circle. The limit $g_{s} \rightarrow 0$ with a fixed $\alpha^{\prime}$ yields the $\mathcal{N}=(2,0)$ little string theory.

The $2 \mathrm{~d}$ gauge theories on IIA little strings at $R_{A} \gg\left(\alpha^{\prime}\right)^{\frac{1}{2}}$ has been studied in [7], in the $6 \mathrm{~d}$ 'Coulomb phase' with nonzero $\phi$, separating all M5-branes along $x^{11}$. [7] discussed it in the context of type IIB strings on $A_{N-1}$ singularity, but let us review it in the M-theory context here. The M-theory branes are shown in figure 1, where the M-theory circle radius is given by $R_{M}=g_{s} \ell_{s}$ (where $\alpha^{\prime}=\ell_{s}^{2}$ ). See also table 2 for coordinates. The tension of the strings is given by $\sim \frac{R_{M}}{\ell_{P}^{3}}=\frac{R_{M}}{g_{s} \ell_{s}^{3}}=\ell_{s}^{-2}$ in the original type IIA string theory, and we are interested in the low energy $2 \mathrm{~d}$ theory at excitation energy $E \ll g_{\mathrm{YM}}$, where $g_{\mathrm{YM}}$ is the $2 \mathrm{~d}$ gauge coupling defined in (3.1). To ease the construction of this theory, we compactify $x^{9}$ direction along a circle with radius $R_{M}^{\prime}$. Since NS5-branes are localized at $x^{9}=0$ and M2branes are attached to them, this compactification cannot be seen by the low energy CFT on the strings, although it will be seen by the UV gauge theory we construct. Now we make a 9-11 flip, regarding $x^{9}$ as the M-theory circle direction. The new type IIA theory would have its own coupling and string scale $g_{s}^{\prime}, \ell_{s}^{\prime}$, satisfying $g_{s}^{\prime} \ell_{s}^{\prime}=R_{M}^{\prime}, g_{s}^{\prime}\left(\ell_{s}^{\prime}\right)^{3}=\ell_{P}^{3}$. The tension of the string given by the D2-branes suspended between NS5-branes is $\frac{R_{M}}{g_{s}^{\prime}\left(\ell_{s}^{\prime}\right)^{3}}=\frac{R_{M}}{\ell_{P}^{3}}=\ell_{s}^{-2}$, same as in the original type IIA picture. Now the low energy $2 d$ theory living on the D2-branes is easy to identify. It is a circular quiver $\mathrm{U}(k)^{N}$ gauge theory with $\mathcal{N}=(4,4)$ supersymmetry [26]. Each gauge node (labeled by $i=1, \cdots, N)$ has vector multiplet fields $A_{\mu}^{(i)}, a_{\alpha \dot{\beta}}^{(i)}$ and fermions, where $\alpha, \dot{\beta}$ are the $\mathrm{SO}(4)=\mathrm{SU}(2)_{L 1} \times \mathrm{SU}(2)_{R 1}$ spinor indices. 
There is a bi-fundamental hypermultiplet mode connecting adjacent gauge nodes. The hypermultiplet fields between $i$ 'th and $i+1^{\prime}$ th node are denoted by complex scalars $\Phi_{A}^{(i)}$ and fermions. Compared to be previous type IIB setting, or the original type IIA setting, in which we had $\mathrm{SO}(4)=\mathrm{SU}(2)_{L 2} \times \mathrm{SU}(2)_{R 2}$ R-symmetry, only the diagonal $\mathrm{SU}(2)_{D}$ survives after the $x^{9}$ circle compactification. So the doublet $A$ index can be regarded as the identification of the previous $a$ and $A$ indices. The $(4,4)$ supercharges are $Q_{\alpha+}^{A}, Q_{\dot{\alpha}-}^{A}$, subject to reality conditions. The $\mathrm{SU}(2)_{D} \mathrm{UV}$ symmetry is supposed to enhance to a full $\mathrm{SO}(4)$ in the IR. This is because the $2 \mathrm{~d}$ IR limit is equivalent to the strong coupling limit, which in M-theory takes $\mathbb{R}^{3} \times S^{1}$ to the large radius limit $\mathbb{R}^{4}$ with $\mathrm{SO}(4)$ symmetry. However, full $\mathrm{SO}(4)$ is invisible in the UV gauge theory. The incapability of seeing the second Cartan of $\mathrm{SO}(4)$ from this UV theory will make it impossible to study the full IR elliptic genus. This will be a motivation to study a $(0,4)$ supersymmetric UV gauge theory for the type IIA little strings, in section 3.1.

The coupling for the $i$ 'th $\mathrm{U}(k)$ gauge field is given by

$$
\frac{1}{g_{Y M, i}^{2}}=\frac{\left(\alpha_{i+1}-\alpha_{i}\right) R_{M} \ell_{s}^{\prime}}{g_{s}^{\prime}}=\frac{\left(\alpha_{i+1}-\alpha_{i}\right) g_{s}^{2} \ell_{s}^{4}}{\left(R_{M}^{\prime}\right)^{2}}
$$

so the coupling becomes large in the little string decoupling limit $g_{s} \rightarrow 0$. We shall study the protected elliptic genera of this system in the weak coupling limit, in the $(0,4)$ supersymmetric version of the gauge theory that will be explained in section 3.1. One can turn on three FI parameters $\zeta_{I}^{(i)}$ for each $\mathrm{U}(k)_{i}$ gauge group, which is a triplet of $\mathrm{SU}(2)_{D}$ rotating 678. This corresponds to the relative position of the $i+1$ 'th NS5-brane from the $i$ 'th NS5-brane along 678 directions. So one obtains the condition $\sum_{i=1}^{N} \zeta_{I}^{(i)}=0$, since one should come back to the original NS5-brane after going around the quiver once.

The gauge theory has $\mathrm{U}(k)^{N}$ Coulomb branch, whose scalars represent the motion of D2-branes along 1234 directions. This would define the Coulomb branch CFT which is relevant for studying the IIA little strings. On the other hand, the $N$ fractional strings suspended between different adjacent pairs of NS5-branes can combine to make a fully winding D2-brane along $x^{11}$, which may leave the NS5-brane along the 6789 directions (among which $x^{9}$ is the circle direction of the M-theory). For instance, at $k=1$, the positions of the D2-branes along 678 is parameterized by the Higgs branch scalars, breaking $\mathrm{U}(1)^{N}$ to $\mathrm{U}(1)$ which lives on the D2-brane separated from the NS5-branes. The U(1) gauge field on this D2 would dualize to a compact scalar, parametrizing the $x^{9}$ circle direction probed by the D2-brane. More precisely, at $k=1$, the vanishing condition of the potential energy is given by

$$
\Phi_{A}^{(i)} a_{\alpha \dot{\beta}}^{(i)}-a_{\alpha \dot{\beta}}^{(i-1)} \Phi_{A}^{(i)}=0, \quad \zeta_{I}^{(i)}+\left(\sigma_{I}\right)_{B}^{A} \Phi_{A}^{(i)} \bar{\Phi}^{B(i)}=\left(\sigma_{I}\right)^{A}{ }_{B} \bar{\Phi}^{B(i-1)} \Phi_{A}^{(i-1)} .
$$

In the Higgs branch, one sets all $a_{\alpha \dot{\beta}}^{(i)}$ 's to be equal, so that the first equation is solved by breaking $\mathrm{U}(1)^{N} \rightarrow \mathrm{U}(1)$. There is always a nonzero solution to the second equation.

Since the Higgs branch now represents the strings leaving the NS5-branes, we are only interested in the Coulomb branch CFT in the IR limit. However, the Higgs branch cannot be detached from the Coulomb branch CFT by any deformation of the theory. This is in 
contrast to the $2 \mathrm{~d}$ gauge theories for the type IIB strings, in which case the Higgs branch CFT of our interest could be detached from the Coulomb branch CFT by turning on $\mathrm{U}(k)$ FI parameters. In fact, with generic FI term $\xi_{I}^{(i)}$, the Coulomb branch will be all lifted as $\mathrm{U}(1)^{N} \rightarrow \mathrm{U}(1)$. Since the elliptic genus formula of [19, 20] is computing the index of CFT with generic nonzero FI parameters, this formula will compute the unwanted Higgs branch index, with lifted Coulomb branch. Apart from the absence of the SU(2) $L 2$ in UV, this is another reason that the above $(4,4) \mathrm{CFT}$ is inconvenient for studying the little string physics.

One can also add fractional D2-branes to this construction. Namely, the number of $i$ 'th D2-branes between $i$ 'th and $i+1$ 'th NS5-branes can be all different, $n_{i}$, forming a circular $\mathrm{U}\left(n_{1}\right) \times \cdots \times \mathrm{U}\left(n_{N}\right)$ quiver.

\section{$3.1 \mathcal{N}=(0,4)$ gauge theory descriptions}

As explained, the $\mathcal{N}=(4,4)$ gauge theories for IIA little strings only see $\mathrm{SU}(2)_{D} \subset \mathrm{SO}(4)$ part of the R-symmetry. Although we expect the symmetry enhancement to happen in IR, this means that the UV gauge theory would be of limited use. Also, studying the spectrum of the Coulomb branch CFT will be difficult with the approaches of $[19,20]$. Closely following the idea of $[15,27]$, we shall engineer $(0,4)$ UV gauge theories for the IIA string systems which resolve all these problems. We also stress that, although the main interest of $[15,27]$ was studying the strings of the $6 \mathrm{~d}$ SCFTs rather than the little strings, figure 6 of [27] does show the $(0,4)$ quiver for the little strings, whose QFT details and physics we shall explain now.

Now on top of the IIA branes explained after the $x^{9}-x^{11}$ flip, we also put one D6-brane extended along 012345,11 and localized at $x^{6}=x^{7}=x^{8}=0$. See table 3. Now with a D6brane uplifting to the Taub-NUT space in M-theory, the SU(2) which rotates 678 directions in weakly coupled type IIA is interpreted differently in the IR CFT in this setting. Namely, the low energy limit of the $2 \mathrm{~d}$ gauge theory is realized by taking the M-theory limit $R_{M}^{\prime} \rightarrow$ $\infty$ (after the 9-11 flip): see (3.1). So the embedding of the UV gauge theory's symmetries into the infrared R-symmetry has to be understood in the $R_{M}^{\prime} \rightarrow \infty$ limit, where we have $\mathbb{R}^{4}$. The $\mathrm{SO}(3)$ rotating the asymptotic $\mathbb{R}^{3}$ of Taub-NUT rotates the $\mathbb{R}^{4}$ as $\mathrm{SU}(2)_{R 2}$ in 'IR.' Also, after compactifying one more circle $x^{5}$, we can turn on a background gauge field of the D6-branes, as $A_{5}^{i}+i A_{11}^{i} \equiv m^{i} \sim(m, 2 m, 3 m, \cdots, N m)$ with nonzero $B_{5,11}$ turned on. The parameter $m$ realizes the chemical potential for the Cartan of SU(2) $)_{L 2}[15,27] .{ }^{2}$ Thus, we can turn on the full set of $\mathrm{SO}(4)_{R}$ chemical potentials of the partition function after $T^{2}$ compactification, in this setting. From the $2 \mathrm{~d}$ gauge theory viewpoint, adding one D6-brane just affects the way we connect the UV regime $\mathbb{R}^{3} \times S^{1}$ at weak-coupling with the IR regime $\mathbb{R}^{4}$ at strong coupling. Since the IR brane configuration is identical to the original M2-M5 system, we expect the $(0,4)$ gauge theory to flow to the same $(4,4)$ CFT on the Coulomb branch. (However, see section 4 for discussions on irrelevant sectors within this gauge theory.)

A $2 \mathrm{~d} \mathcal{N}=(0,4) \mathrm{UV}$ gauge theory is engineered from this brane setting, with supercharges given by $Q^{A \dot{\alpha}}$. The fields can be characterized again by a circular quiver of

\footnotetext{
${ }^{2}$ Note that $m$ is turned on only after compactifying the $2 \mathrm{~d}$ gauge theory on $S^{1}$ or $T^{2}$. So whenever we address $(0,4)$ or $(4,4)$ SUSY later, it holds for the QFT on $\mathbb{R}^{1,1}$ with $m=0$. The SUSY preserved after $T^{2}$ compactication with various chemical potentials turned on is summarized in table 1 of [15].
} 


\begin{tabular}{|c|c|c|c|c|c|c|c|c|c|c|}
\hline & $x^{0}$ & $x^{1}$ & $x^{2}$ & $x^{3}$ & $x^{4}$ & $x^{5}$ & $x^{6}$ & $x^{7}$ & $x^{8}$ & $x^{11}\left(S^{1}\right)$ \\
\hline$N$ NS5 & $\times$ & $\times$ & $\times$ & $\times$ & $\times$ & $\times$ & & & & $\alpha_{i}$ \\
\hline$n_{i}$ D2 & $\times$ & & & & & $\times$ & & & & $\left(\alpha_{i}, \alpha_{i+1}\right)$ \\
\hline 1 D6 & $\times$ & $\times$ & $\times$ & $\times$ & $\times$ & $\times$ & & & & $\times$ \\
\hline
\end{tabular}

Table 3. Brane construction of $2 \mathrm{~d} \mathcal{N}=(0,4)$ gauge theory.

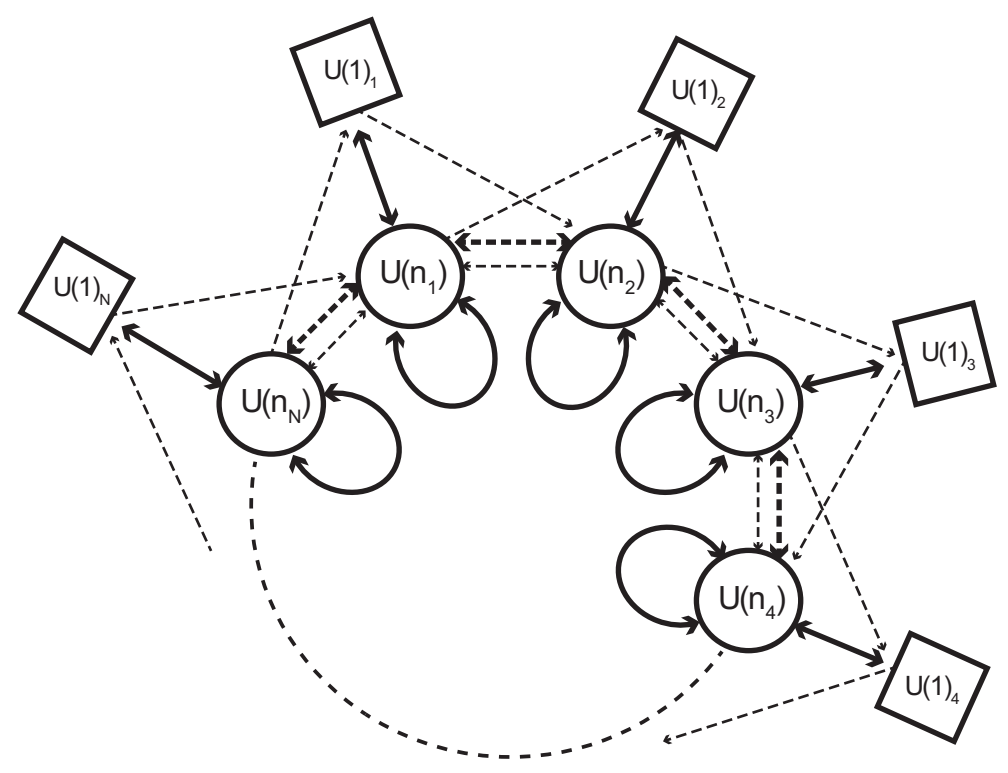

Figure 2. $\hat{A}_{N-1}$ quiver diagram of the $2 \mathrm{~d} \mathcal{N}=(0,4)$ gauge theory for the IIA strings. Solid lines denote the hypermultiplets, thin dashed lines denote the Fermi multiplets, and thick dashed lines denote the twisted hyper multiplets.

\begin{tabular}{|c|c|c|c|}
\hline Multiplet & Fields & $\mathrm{U}\left(n_{i}\right)$ & $\mathrm{U}(1)_{m}$ \\
\hline Vector & $A_{\mu}^{(i)}, \bar{\lambda}_{+}^{(i) A \dot{\alpha}}$ & $\operatorname{adj}_{i}$ & 0 \\
\hline Hyper & $q_{\dot{\alpha}}^{(i)}, \psi_{-}^{(i) A}$ & $\mathbf{n}_{i}$ & 0 \\
\hline Hyper & $a_{\alpha \dot{\beta}}^{(i)}, \lambda_{\alpha-}^{(i) A}$ & $\operatorname{adj}_{i}$ & 0 \\
\hline Twisted hyper & $\Phi_{A}^{(i)}, \Psi_{-}^{(i) \dot{\alpha}}$ & $\left(\mathbf{n}_{i-1}, \overline{\mathbf{n}}_{i}\right)$ & 1 \\
\hline Fermi & $\Psi_{\beta+}^{(i)}$ & $\left(\mathbf{n}_{i-1}, \overline{\mathbf{n}}_{i}\right)$ & 1 \\
\hline Fermi & $\psi_{+}^{(i)}$ & $\mathbf{n}_{i}$ & 1 \\
\hline Fermi & $\tilde{\psi}_{+}^{(i)}$ & $\overline{\mathbf{n}}_{i}$ & -1 \\
\hline
\end{tabular}

Table 4. Fields of the $\mathcal{N}=(0,4)$ quiver gauge theory. 
figure 2. See also figure 6 of [27], in which this quiver was first discovered. Each circular node involves $\mathcal{N}=(0,4) \mathrm{U}\left(n_{i}\right)$ gauge multipletiplet $\left(A_{\mu}, \bar{\lambda}_{+}^{A \dot{\alpha}}\right)$, and a $\mathcal{N}=(0,4)$ adjoint hypermultiplet $\left(a_{\alpha \dot{\beta}}, \lambda_{\alpha-}^{A}\right)$, denoted by the solid lines. $n_{i}$ 's are the number of the D2-branes suspended between adjacent NS5-branes. Thick dashed lines between two circular nodes denote the bi-fundamental twisted hypermultiplets $\left(\Phi_{A}, \Psi_{-}^{\dot{\alpha}}\right)$. Thin dashed lines between two circular nodes denote the bi-fundamental fermi multiplets $\Psi_{\beta+}$. D6-brane introduces extra fields: fundamental hyper multiplets $\left(q_{\dot{\alpha}}, \psi_{-}^{A}\right)$ and Fermi multiplets $\psi_{+}, \tilde{\psi}_{+}$. These fields are are summarized in table 4 . As explained in the previous paragraph, and just like $\left[15,27\right.$, the chemical potentials for $\mathrm{U}(1)_{i}$ and $\mathrm{U}(1)_{i+1}$ are locked as $m_{i+1}-m_{i}=m$, so that one just has one $\mathrm{U}(1)_{m}$. Compared to the previous $(4,4)$ gauge theory for the IIA strings, the $(0,4)$ fields on the first and third lines of table 4 are forming the $(4,4)$ vector multiplet, which we decomposed as above since the system does not preserve $(4,4)$ SUSY. Also, the fields on the fourth and fifth lines form the previous $(4,4)$ hypermultiplet. They again make a twisted Higgs branch, which represents the degrees of freedom of fully winding D2-branes leaving the NS5-branes. The Coulomb branch of the $(4,4)$ theory is replaced here by the Higgs branch formed by the second and third lines, which is our main interest when we study the IIA little strings.

The SUSY action of the $(0,4)$ gauge theory can also be easily constructed. From the $(0,2)$ supersymmetric formalism, one has to determine the holomorphic potentials $E_{\Psi}, J_{\Psi}$ for each Fermi multiplet $\Psi$, ensuring the $(0,4)$ SUSY enhancement. For instance, see [28] for how this can be done. Here, following [28], we simply write down these potentials for our theory. Let us call the $(0,2)$ Fermi multiplet from the $(0,4)$ vector multiplet as $\Lambda_{i}$, which is made of $\bar{\lambda}^{11}$ and $\bar{\lambda}^{2 \dot{2}}$. Then one should first take

$$
J_{\Lambda_{i}}=q_{i} \tilde{q}_{i}+\left[B_{i}, \tilde{B}_{i}\right]-\xi_{\mathbb{C}}, \quad E_{\Lambda_{i}}=\Phi_{i+1} \tilde{\Phi}_{i+1}-\tilde{\Phi}_{i} \Phi_{i}
$$

for $(0,4)$ SUSY [28]. Here and below, we use the chiral superfield notation $q_{\dot{\alpha}}=\left(q, \tilde{q}^{\dagger}\right)$, $a_{1 \dot{\beta}}=\left(B, \tilde{B}^{\dagger}\right), \Phi_{A}=\left(\Phi, \tilde{\Phi}^{\dagger}\right)$ for a while. We also inserted the FI parameter $\xi_{\mathbb{C}}$ for later use, which corresponds to turning on worldvolume $B_{\mu \nu}$ field on 1234 directions. The above $J, E$ should be accompanied by other $J, E$ functions for other Fermi fields, to satisfy $\sum_{\Psi} E_{\Psi} J_{\Psi}=0$ after summing over all Fermi multiplets $\Psi$. This is another requirement from SUSY. To meet the last condition, one should turn on the following potentials for other Fermi multiplet fields:

$$
\begin{array}{ll}
E_{\tilde{\psi}_{i}}=\tilde{q}_{i-1} \Phi_{i}, & J_{\tilde{\psi}_{i}}=-\tilde{\Phi}_{i} q_{i-1}, \quad E_{\psi_{i}}=\Phi_{i+1} q_{i+1}, \quad J_{\psi_{i}}=\tilde{q}_{i+1} \tilde{\Phi}_{i+1} \\
E_{\Psi_{i}}=\Phi_{i} B_{i}-B_{i-1} \Phi_{i}, & J_{\Psi_{i}}=\tilde{B}_{i} \tilde{\Phi}_{i}-\tilde{\Phi}_{i} \tilde{B}_{i-1} \\
E_{\tilde{\Psi}_{i}}=\tilde{B}_{i-1} \Phi_{i}-\Phi_{i} \tilde{B}_{i}, & J_{\tilde{\Psi}_{i}}=B_{i} \tilde{\Phi}_{i}-\tilde{\Phi}_{i} B_{i-1}
\end{array}
$$

The bosonic potential is $V=2 \sum_{\Psi}\left(\left|J_{\Psi}\right|^{2}+\left|E_{\Psi}\right|^{2}\right)+\frac{1}{2} \sum_{i} D_{i}^{2}$ with $D_{i}$ given by ${ }^{3}$

$$
D_{i}=q_{i} q_{i}^{\dagger}-\tilde{q}_{i}^{\dagger} \tilde{q}_{i}+\left[B_{i}, B_{i}^{\dagger}\right]+\left[\tilde{B}_{i}, \tilde{B}_{i}^{\dagger}\right]-\Phi_{i}^{\dagger} \Phi_{i}+\tilde{\Phi}_{i} \tilde{\Phi}_{i}^{\dagger}+\Phi_{i+1} \Phi_{i+1}^{\dagger}-\tilde{\Phi}_{i+1}^{\dagger} \tilde{\Phi}_{i+1}-\xi_{\mathbb{R}}
$$

\footnotetext{
${ }^{3}$ The factor 2 in front of $\left|J_{\Psi}\right|^{2}+\left|E_{\Psi}\right|^{2}$ has to be included if we follow the convention of [28] for (3.3), (3.4).
} 
After some rearrangement, one obtains

$$
\begin{gathered}
V=\sum_{i=1}^{N}\left[\frac{1}{2}\left(q_{i \alpha}\left(\sigma^{m}\right)^{\dot{\alpha}}{ }_{\dot{\beta}} \bar{q}_{i}^{\dot{\beta}}+\frac{1}{2}\left(\sigma^{m}\right)^{\dot{\alpha}}{ }_{\dot{\beta}}\left[a_{i \alpha \dot{\alpha}}, a_{i}^{\alpha \dot{\beta}}\right]-\xi^{m}\right)^{2}+\left(\left(\sigma^{I}\right)^{A}{ }_{B} \Phi_{i A} \bar{\Phi}_{i}^{B}-\left(\sigma^{I}\right)^{A}{ }_{B} \bar{\Phi}_{i-1}^{B} \Phi_{i-1, A}\right)^{2}\right. \\
\left.+\left|\Phi_{i A} q_{i \dot{\alpha}}\right|^{2}+\left|\Phi_{i+1, A}^{\dagger} q_{i \dot{\alpha}}\right|^{2}+\left|\Phi_{i A} a_{i \alpha \dot{\beta}}-a_{i-1, \alpha \dot{\beta}} \Phi_{i A}\right|^{2}\right]
\end{gathered}
$$

where $\xi^{3}=\xi_{\mathbb{R}}$ and $\xi^{1}+i \xi^{2} \sim \xi_{\mathbb{C}}$, with manifest $\mathrm{SU}(2)_{R 1} \times \mathrm{SU}(2)_{R 2}=\mathrm{SO}(4)_{R}$ symmetry for the $(0,4)$ theory.

Note that with nonzero $\xi^{m}, q_{i \dot{\alpha}}$ fields should be nonzero at low energy, which lift the twisted Higgs branch of $\Phi_{i A}$. Namely, even if $n_{1}=n_{2}=\cdots=n_{N}$, they cannot combine and leave the NS5-branes unlike the $\mathcal{N}=(4,4)$ model. Also, the previous $(4,4)$ Coulomb branch fields $a_{\alpha \dot{\beta}}$ form $(0,4)$ Higgs branch fields, together with new fields $q_{\dot{\alpha}}$. The $(0,4)$ setting will thus be computing the correct little string elliptic genus. However, the $(0,4)$ elliptic genus will also capture a subtle trace of the presence of a D6-brane from the sector with $n_{1}=n_{2}=\cdots=n_{N}$, in which case the D2-branes make full windings along $x^{11}$. This can be easily accounted for and factored out, after which we shall be obtaining the IIA little string index. We shall explain this in section 4 .

\subsection{The elliptic genus of IIA little strings}

We define the index of IIA little string theory wrapping a spatial circle along $x^{5}$, as follows,

$$
Z_{\mathrm{IIA}}\left(\alpha_{i}, \epsilon_{ \pm}, m ; q^{\prime}, w^{\prime}\right)=\operatorname{Tr}\left[(-1)^{F} q^{\prime H_{L}} \bar{q}^{\prime H_{R}} w^{\prime k} e^{2 \pi i \alpha_{i} \Pi_{i}} e^{2 \pi i \epsilon_{-}\left(2 J_{L 1}\right)} e^{2 \pi i m\left(2 J_{2 L}\right)} e^{2 \pi i \epsilon_{+}\left(2 J_{1 R}+2 J_{2 R}\right)}\right] .
$$

$\Pi_{i}$ are charges of the self-dual tensor fields, supported on each M5-brane, with the chemical potentials, $\alpha_{i}$. $q^{\prime}$ are the fugacity variable for the momentum, and $w^{\prime}$ the winding fugacity of the IIA little strings. $2 \pi \alpha_{i} R_{M}$ are the positions of the 5 -branes along $x^{11} . Z_{I I A}$ is the BPS partition function of the little string theory on Omega-deformed $\mathbb{R}^{4} \times T^{2}$.

An M2-brane suspended between the $i$ 'th interval between the M5-branes, $\left(\alpha_{i}, \alpha_{i+1}\right)$, carries nonzero charges $\Pi_{i}=1$ and $\Pi_{i+1}=-1$. The charges $e_{i}-e_{i+1}$ form the simple roots of the $A_{N-1}$ algebra, for $i=1, \cdots, N-1$. Finally, the charge $e_{N}-e_{1}$, accompanied by an extra winding $k=1$ on the $x^{11}$ circle, combines with the $A_{N}$ simple roots to make the simple roots of $\hat{A}_{N-1}$. The fugacity variables corresponding to these simple roots are given by

$$
v_{1} \equiv e^{2 \pi i \alpha_{12}}, \quad v_{2} \equiv e^{2 \pi i \alpha_{23}}, \cdots, v_{N-1} \equiv e^{2 \pi i \alpha_{N-1, N}}, \quad v_{N} \equiv e^{2 \pi i \alpha_{N, N+1}}=e^{2 \pi i \alpha_{N, 1}} w^{\prime} .
$$

where $\alpha_{i j}=\alpha_{i}-\alpha_{j}$. For convenience, we introduce $\alpha_{N+1}$, where $e^{-2 \pi i \alpha_{N+1}}=e^{-2 \pi i \alpha_{1}} w^{\prime}$.

For large $R_{A}$, the low energy degrees of freedom living on the winding strings decouple from the $6 \mathrm{~d}$ degrees on the 5 -branes. So the index of the IIA little string theory on $\mathbb{R}^{4} \times T^{2}$ factorizes as

$$
Z_{\mathrm{IIA}}\left(\alpha_{i}, \epsilon_{ \pm}, m ; q^{\prime}, w^{\prime}\right)=Z_{\mathrm{mom}}^{\mathrm{IIA}}\left(\epsilon_{ \pm}, m ; q^{\prime}\right) Z_{\mathrm{string}}^{\mathrm{IIA}}\left(\alpha_{i}, \epsilon_{ \pm}, m ; q^{\prime}, w^{\prime}\right) .
$$

$Z_{\text {mom }}^{\text {IIA }}$ comes from the Abelian tensor theories on $N$ separated M5-branes wrapping a spatial circle, and carries only $H_{L}$ charge in the index but carries $k=0$. Unlike the IIB 
perturbative index $Z_{\text {pert }}^{\mathrm{IIB}}$ which had massive W-boson contributions, the IIA 5-brane does not have extra massive particle states in it (because it only has strings). This contribution factorizes into $N$ single 5-brane contributions. It can be computed either from $N$ Abelian tensor multiplet, or equivalently from the multiple D0-brane index bound to a single D4-brane [22]. The result is

$$
Z_{\mathrm{mom}}^{\mathrm{IIA}}\left(\epsilon_{ \pm}, m ; q^{\prime}\right)=P E\left[N I_{-}\left(\epsilon_{1,2}, m\right) \frac{q^{\prime}}{1-q^{\prime}}\right]
$$

where

$$
I_{-}\left(\epsilon_{1,2}, m\right) \equiv \frac{\sinh \frac{2 \pi i\left(m+\epsilon_{-}\right)}{2} \sinh \frac{2 \pi i\left(m-\epsilon_{-}\right)}{2}}{\sinh \frac{2 \pi i \epsilon_{1}}{2} \sinh \frac{2 \pi i \epsilon_{2}}{2}}
$$

with $\epsilon_{ \pm}=\frac{\epsilon_{1} \pm \epsilon_{2}}{2}[22]$.

The contribution $Z_{\text {string }}^{\text {IIA }}$ comes from the wrapped IIA strings. It can be computed from the elliptic genus of the $2 \mathrm{~d}(4,4)$ SCFT in the Coulomb branch. Since this direct approach is difficult, we use the elliptic genera of the $(0,4)$ gauge theories that we have explained in the previous subsection. Let us call the elliptic genus of the $\prod_{i=1}^{N} \mathrm{U}\left(n_{i}\right)$ gauge theory as $Z_{\text {string }}^{\left(n_{1}, \cdots, n_{N}\right)}\left(\epsilon_{ \pm}, m ; q^{\prime}\right)$, and also define

$$
\begin{aligned}
\hat{Z}_{\text {string }}^{\mathrm{IIA}}\left(\alpha_{i}, \epsilon_{ \pm}, m ; q^{\prime}, w^{\prime}\right) & \equiv \sum_{n_{i}=0}^{\infty} e^{2 \pi i \sum_{i=1}^{N} n_{i} \alpha_{i, i+1}} Z_{\text {string }}^{\left(n_{1}, \ldots, n_{N}\right)}\left(\epsilon_{ \pm}, m ; q^{\prime}\right) \\
& =\sum_{n_{i}=0}^{\infty}\left(v_{1}\right)^{n_{1}}\left(v_{2}\right)^{n_{2}} \cdots\left(v_{N}\right)^{n_{N}} Z_{\text {string }}^{\left(n_{1}, \ldots, n_{N}\right)}\left(\epsilon_{ \pm}, m ; q^{\prime}\right)
\end{aligned}
$$

Naively, one would expect $Z_{\text {string }}^{\text {IIA }}=\hat{Z}_{\text {string }}^{\text {IIA }}$, but we discuss their relations in detail at the beginning of section 4 . Here, let us just explain how to compute $\hat{Z}_{\text {string }}^{\text {IIA }}$.

Applying the contour integral formula for elliptic genus in [20], one should study

$$
\begin{aligned}
\hat{Z}_{\text {string }}^{\left(n_{1}, \cdots, n_{N}\right)} & =\oint\left[\prod_{i=1}^{N} \frac{1}{n_{i} !} \prod_{I_{i}=1}^{n_{i}} \frac{2 \pi^{2} \eta^{2} d u_{I_{i}}}{2 \pi i}\right] \prod_{i=1}^{N} Z_{\text {vec }}^{(i)} \cdot \prod_{i=1}^{N} Z_{\text {hyper }}^{(i, i+1)} \\
Z_{\text {vec }}^{(i)} & =\frac{\prod_{I \neq J} \theta_{1}\left(u_{I J}\right) \prod_{I, J=1}^{n_{i}} \theta_{1}\left(u_{I J}+2 \epsilon_{+}\right)}{\prod_{I=1}^{n_{i}} \theta_{1}\left(\epsilon_{+} \pm u_{I}\right) \prod_{I, J=1}^{n_{i}} \theta_{1}\left(\epsilon_{1,2}+u_{I J}\right)} \\
Z_{\text {hyper }}^{(i, i+1)} & =\frac{\prod_{I=1}^{n_{i}} \theta_{1}\left(m+u_{I}\right) \prod_{J=1}^{n_{i}+1} \theta_{1}\left(m-u_{J}\right) \prod_{I=1}^{n_{i}} \prod_{J=1}^{n_{i+1}} \theta_{1}\left(m-\epsilon_{-}+u_{I J}\right) \theta_{1}\left(-m-\epsilon_{-}+u_{J I}\right)}{\prod_{I=1}^{n_{i}} \prod_{J=1}^{n_{i}+1} \theta_{1}\left( \pm m-\epsilon_{+}+u_{I J}\right)}
\end{aligned}
$$

where $i=N+1$ by definition means $i=1$ (from the circular nature of the quiver). Apparently, it may look that this contour integral is much more complicated than (2.6). But one can use the analysis of (2.6) which led to (2.7), to get a closed form expression of (3.13).

To explain the simple structure of this contour integral, let us start by noting a $6 \mathrm{~d}$ interpretation of our 2d gauge theories. From the D2-D6 engineering of this system, one can regard the D2-branes as the instanton strings of the 6d SYM theories living on D6-branes. Namely, one starts from the $6 \mathrm{~d} \mathcal{N}=1$ gauge theory with $\mathrm{U}(1)^{N}=\prod_{i=1}^{N} \mathrm{U}(1)_{i}$ gauge group, which form a circular quiver by having $6 \mathrm{~d}$ bi-fundamental hypermultiplets in $\mathrm{U}(1)_{i} \times$ $\mathrm{U}(1)_{i+1}$. The instanton string number in $\mathrm{U}(1)_{i}$ gauge group is $n_{i}$. Of course, from the viewpoint of field theory solitons, $\mathrm{U}(1)$ instantons are singular but this is naturally included 
in the instanton counting, which is precisely what we mean by the $\mathrm{U}(1)$ instantons here. Then our $2 \mathrm{~d}(0,4)$ quiver is the ADHM gauge theory for these $\mathrm{U}(1)^{N}$ instanton strings.

Now comes an important observation, that our $\mathrm{U}(1)^{N}$ gauge theory can be embedded into the $6 \mathrm{~d} \mathrm{U}(N)$ maximal SYM, by keeping the diagonal modes in the $\mathcal{N}=1$ vector multiplet and only keeping the 'next-to-diagonal' modes in the $\mathcal{N}=1$ adjoint hypermultiplet. Namely, calling the $6 \mathrm{~d}$ hypermultiplet field $\Phi$, given by an $N \times N$ matrix, the modes which are kept are

$$
\Phi=\left(\begin{array}{cccccc}
0 & \Phi_{1,2} & \cdots & & & \Phi_{1, N} \\
\Phi_{2,1} & 0 & \Phi_{2,3} & \cdots & & \\
0 & \Phi_{3,2} & 0 & & & \\
& & & & \\
\vdots & & & & 0 & \Phi_{N-1, N} \\
\Phi_{N, 1} & & & & \Phi_{N, N-1} & 0
\end{array}\right)
$$

i.e. $\Phi_{i, i+1} \neq 0$ and $\Phi_{i, i-1} \neq 0$ with $i=N+1 \sim 1$ understood. The measures $\prod_{i=1}^{N} Z_{\text {vec }}^{(i)}$. $\prod_{i=1}^{N} Z_{\text {hyper }}^{(i, i+1)}$ in (3.13) are precisely the 1-loop determinants for the instanton zero modes caused by the $6 \mathrm{~d}$ modes kept in the trunctation of the $6 \mathrm{~d} \mathrm{U}(N)$ theory, after plugging in $\alpha_{i}=0$ in the integrand appearing in (2.6).

With this observation, all the simplifications that happened to get (2.7) apply to our IIA elliptic genera as well. First of all, classifying the poles with nonzero JK-Res following [21], one again finds that no poles are to be kept from $Z_{\text {hyper }}^{(i, i+1)}$. This is a simple consequence of the cancelation mentioned in the last paragraph of p.6, which applies completely within the modes that we keep in the truncation. Then, the poles come only from $Z_{\text {vec }}^{(i)}$. Since each $Z_{\text {vec }}^{(i)}$ is completely factorized with other vector multiplet determinants $Z_{\text {vec }}^{(j)}$ with $j \neq i$, the pole locations for $u_{I_{i}}$ are simply those for the $n_{i} \mathrm{U}(1)$ instanton contour integral. So at each $i$, the poles with nonzero JK-Res are labeled by a Young diagram with $n_{i}$ boxes, which we call $Y_{i}$. So the poles of our $2 \mathrm{~d} \prod_{i=1}^{N} \mathrm{U}\left(n_{i}\right)$ gauge theory's elliptic genus are classified by $N$ Young diagrams, $\left\{Y_{1}, \ldots, Y_{N}\right\}$, where $\left|Y_{i}\right|=n_{i}$. Now we consider the residue at a given pole, labeled by $\left\{Y_{i}\right\}$. The cancelation of $\theta_{1}$ functions in (2.6) for the mass-deformed maximal SYM, again happens in our case with the above truncation. So one just needs to truncate the factors in (2.7). Firstly, $Z_{\mathrm{vec}}^{(i)}$ factor has large cancelations, after which only bosonic modes remain. The remaining factors can be obtained from the denominator of (2.7), by keeping the modes with $i=j$ only in the product $\prod_{i, j=1}^{N}$. Similarly, the measures coming from the $\Phi_{i, i \pm 1}$ modes in (3.14) can also be obtained by suitably truncating the factors in the numerator of (2.7). Namely, the factor $\theta_{1}\left(q ; E_{i j}+m-\epsilon_{-}\right)$in the numerator of (2.7) should be truncated to $j=i+1$, and the factor $\theta_{1}\left(q ; E_{i j}-m-\epsilon_{-}\right)$should be truncated to $j=i-1$, from (3.14). Collecting the truncated modes only, one obtains

$$
Z_{\text {string }}^{\left(n_{1}, \ldots, n_{N}\right)}\left(\epsilon_{ \pm}, m ; q^{\prime}\right)=\sum_{\left\{Y_{1}, \ldots, Y_{N}\right\} ;\left|Y_{i}\right|=n_{i}} \prod_{i=1}^{N} \prod_{(a, b) \in Y_{i}} \frac{\theta_{1}\left(q^{\prime} ; E_{i, i+1}^{(a, b)}-m+\epsilon_{-}\right) \theta_{1}\left(q^{\prime} ; E_{i, i-1}^{(a, b)}+m+\epsilon_{-}\right)}{\theta_{1}\left(q^{\prime} ; E_{i, i}^{(a, b)}+\epsilon_{1}\right) \theta_{1}\left(q^{\prime} ; E_{i, i}^{(a, b)}-\epsilon_{2}\right)},
$$


where

$$
E_{i j}^{(a, b)}=\left(Y_{i, a}-b\right) \epsilon_{1}-\left(Y_{j, b}^{T}-a\right) \epsilon_{2}, \quad E_{i, N+1}^{(a, b)}=E_{i, 1}^{(a, b)} .
$$

$(a, b)$ denotes the position of each box in a Young diagram. $Y_{a, i}$ is the length of the $a^{\prime}$ th row of the Young diagram $Y_{i} . Y_{a, i}^{T}$ is the length of the $a^{\prime}$ th column of $Y_{i}$. Note that $Y_{i, a}-b=$ $h_{i}(s)$ and $Y_{j, b}^{T}-b=v_{j}(s)$ from the definitions of $h_{i}(s)$ and $v_{j}(s)$ given below (2.8). Also, at $\alpha_{i}=\alpha_{j}=0$ in (2.8), $E_{i j}$ reduces to $-E_{i, j}^{(a, b)}$ used in (3.15), with $E_{i j}^{(a, b)}$ defined by (3.16). (Various -1 factors appearing by rewriting $\theta_{1}\left(q^{\prime} ;-z\right)=-\theta_{1}\left(q^{\prime} ; z\right)$ cancel between the numerator and denominator.) This proves that the JK-Res sum of (3.13) yields (3.15).

Naively, one might think that $\hat{Z}_{\text {string }}^{\mathrm{IIA}}$ computed from the $(0,4)$ gauge theory is same as $Z_{\text {string. }}^{\mathrm{IIA}}$. As we emphasized earlier in this section, the contribution from the $(4,4)$ Higgs branch (or the $(0,4)$ twisted Higgs branch) formed by $\Phi_{A}^{(i)}$ is not completely decoupled. We shall explain at the beginning of section 4 what kind of unwanted contribution we expect to get from this extra sector, and proceed our studies with this extra factor divided out in the following subsections.

\section{T-duality of protected little string spectra}

The IIB little string theory on a circle is supposed to be T-dual to IIA little string theory on the dual circle, with the radiii related by $R_{\mathrm{A}}=\frac{\alpha^{\prime}}{R_{\mathrm{B}}}$. The winding IIB little strings on $S_{\mathrm{B}}^{1}$ is dual to the momentum on $S_{\mathrm{A}}^{1}$, and vice versa. Their BPS masses agree with each other, since

$$
m_{\mathrm{IIB} \text { winding }}=\frac{2 \pi R_{\mathrm{B}}}{2 \pi \alpha^{\prime}}=\frac{R_{\mathrm{B}}}{\alpha^{\prime}}=\frac{1}{R_{\mathrm{A}}}=m_{\mathrm{IIA} \text { momentum }} .
$$

The fractional momenta (i.e. $\mathrm{U}(N)$ electric charges) of IIB little string theory are dual to the fractional winding numbers of IIA little strings,

$$
m_{\text {IIB KK }}=\frac{\alpha_{i, i+1}}{R_{\mathrm{B}}} \stackrel{\mathrm{T}-\text { dual }}{\longrightarrow}\left(\alpha_{i, i+1}\right) \frac{R_{\mathrm{A}}}{\alpha^{\prime}}=\alpha_{i, i+1}\left(2 \pi R_{\mathrm{A}}\right) T_{\mathrm{F} 1},
$$

where $\alpha_{i j}=\alpha_{i}-\alpha_{j}$. T-duality between two little string theories is demonstrated by figure 3 .

T-duality between IIA and IIB little string theories implies

$$
\left.Z_{\mathrm{IIA}}\left(\alpha_{i}, \epsilon_{ \pm}, m ; q^{\prime}, w^{\prime}\right)\right|_{q^{\prime} \rightarrow w, w^{\prime} \rightarrow q}=Z_{\mathrm{IIB}}\left(\alpha_{i}, \epsilon_{ \pm}, m ; q, w\right) .
$$

As we stated at the end of section 3.1 , if we use an alternative $2 \mathrm{~d}(0,4)$ gauge theory to compute the elliptic genera $\hat{Z}_{\text {string, }}^{\text {IIA }}$, we shall find a subtle trace of the fact that we made a UV deformation of the gauge theory by putting an extra D6-brane. A spectrum change will happen in a sector with full wound D2-branes along $x^{11}$, namely with states carrying the factors of fugacities $w^{\prime}$ but not $\alpha_{i}^{\prime}$ 's. Let us first explain this small subtlety in our IIA calculation, clarifying how to obtain $Z_{\text {string }}^{\mathrm{IIA}}$ from $\hat{Z}_{\text {string }}^{\mathrm{IIA}}$.

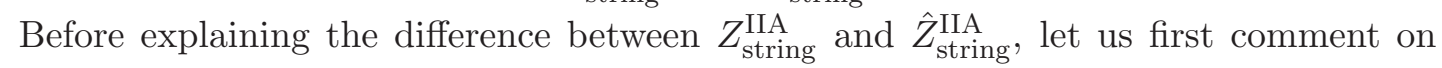
how we first discovered it, which may help the readers to better understand our following explanations. We have first compared $Z_{\mathrm{IIB}}$ and $Z_{\text {mom }}^{\mathrm{IIA}} \hat{Z}_{\text {string }}^{\mathrm{IIA}}\left(\right.$ instead of $\left.Z_{\mathrm{IIA}}=Z_{\text {mom }}^{\mathrm{IIA}} Z_{\text {string }}^{\mathrm{IIA}}\right)$ 

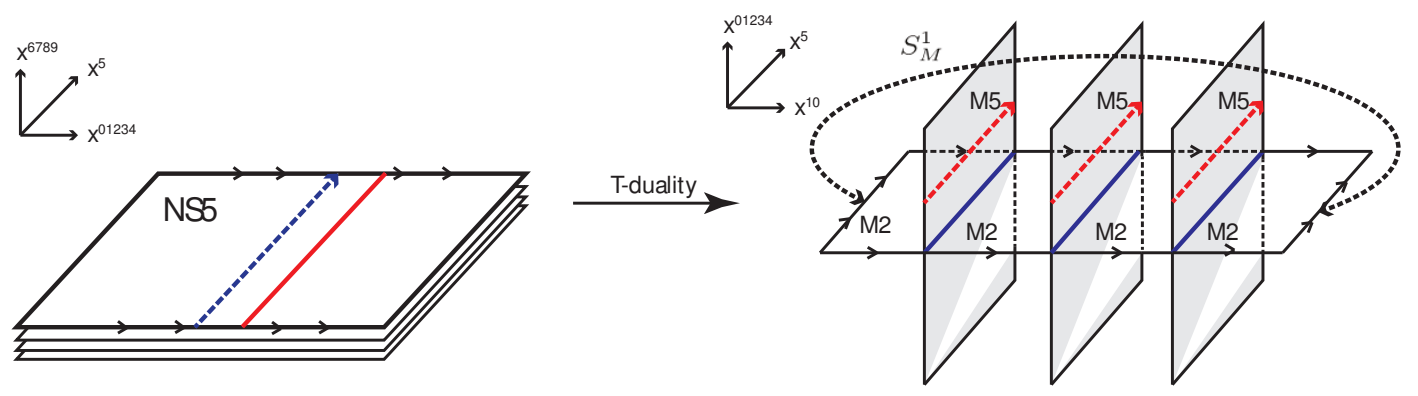

Figure 3. T-duality and M-theory uplift of the IIB setting. The solid lines represent the winding little strings. The dashed lines represent the momentum along the circle.

and found that a small discrepancy happens in a subsector at $w^{0}$ order (with zero winding of IIB strings), and also with no $\mathrm{SU}(N)$ charges, in the type IIB side. Namely, this is a particular subsector of perturbative states captured by $Z_{\text {pert }}^{\text {IIB }}$. Equivalently, on the IIA side, this sector is in the $\left(q^{\prime}\right)^{0}$ order at zero momentum, with $n_{1}=n_{2} \cdots=n_{N}$. Since we empirically found subtle difference only in this sector, we decided to directly count the BPS wavefunctions in this subsector to fully derive this difference. On the IIB side, there is no subtlety in our gauge theory description. The index in the sector with zero $\operatorname{SU}(N)$ electric charges (no dependence on $\alpha_{i}$ ) and zero winding ( $w^{0}$ order) can be extracted from $Z_{\text {pert }}^{\mathrm{IIB}}$ factor. In particular, it comes from the $N$ Cartan modes of the $6 \mathrm{~d} \mathrm{U}(N) \mathrm{SYM}$. Their partition function is given by

$$
P E\left[N I_{+}\left(\epsilon_{1,2}, m\right) \frac{q}{1-q}\right]
$$

In the IIA dual language, the expected T-dual result is obtained by replacing $q \rightarrow w^{\prime}$.

Now let us consider the corresponding sector in the IIA side, at $\left(q^{\prime}\right)^{0}$ order, and also with fully wound D2-branes along $x^{11}$ with $n_{1}=n_{2}=\cdots=n_{N}$. The indices are computed after turning on nonzero FI parameters $\xi^{m}$ with one D6-brane, while the actual spectrum should be studied at $\xi^{m}=0$ without D6-brane whenever a subtlety arises. We study the spectrum directly in both cases, in the subsector specified above, to find the relation between $\hat{Z}_{\text {string }}^{\mathrm{IIA}}$ and $Z_{\text {string }}^{\mathrm{IIA}}$. We first explain the qualitative reason of why the subtlety arises, and then proceed to make a detailed calculation. At $\xi^{m}=0$ in section 3.1, there is an extra twisted Higgs branch which meets the Higgs branch of our interest. The former sector will represent the little strings leaving NS5-branes. The two sectors would decouple in IR, but the $2 \mathrm{~d}$ gauge theory contains both in its Hilbert space. Now by turning on the FI term $\xi^{m}$, the continuum of twisted Higgs branch will be lifted. However, after turning on $\xi^{m}$, it often happens that there appear extra bound states of the continuum with the remaining $2 \mathrm{~d}$ strings of our interest. For instance, see [21] and references therein for many occasions in which extra bound states occur at nonzero FI parameters. Another potential reason for discrepancies between $Z_{\text {string }}^{\mathrm{IIA}}$ and $\hat{Z}_{\text {string }}^{\mathrm{IIA}}$ is that our gauge theories have one D6-brane inserted. When the D2-branes are not leaving NS5-branes, its effect is naturally 
expected to be absent in IR, or equivalently at strong coupling when a D6-brane uplifts to flat spacetime. However, when the branes can leave NS5-branes, the presence of a D6 at $\xi^{m} \neq 0$ will leave a subtle effect as we shall see.

We first consider the case with $\xi^{m}=0$, without a D6-brane. In the sector with $n_{1}=$ $\cdots=n_{N} \equiv n$ that we are considering, the D2-branes can form $n$ full winding branes. So the strings have the right quantum number to leave the NS5-branes. We weight $n$ windings by $\left(w^{\prime}\right)^{n}$, and relax the constraint on fixed $n$. We would like to count the BPS bounds of these strings with $N$ NS5-branes directly, not using the elliptic genus formula of [19, 20]. For convenience, we T-dualize along the $x^{11}$ circle, and obtain many D1-branes along $05, \mathrm{~N}$ centered Taub-NUT on 678 and 11 circle. Firstly, any number $n$ of wrapped D1-branes can form a bound state of multiply wound single string. For each massive particle of this sort, we study its ground state wavefunction on the $N$-centered Taub-NUT. This space has $N$ normalizable harmonic forms, so that there could be $N$ possible bound states of the original $N$ NS5-branes with this particle. The index for this particle is thus $N I_{+}\left(\epsilon_{1,2}, m\right)\left(w^{\prime}\right)^{n}$, where $N$ comes from $N$ normalizable harmonic forms. The $I_{+}$factor appears because this is exactly the same type of bound states as the half-BPS W-bosons in SYM, as in the IIB setting. Summing over $n$ and considering the multi-particle Hilbert space, one obtains

$$
P E\left[N I_{+}\left(\epsilon_{1,2}, m\right) \frac{w^{\prime}}{1-w^{\prime}}\right] \text {. }
$$

This is (4.3), with $q$ replaced by $w^{\prime}$. So the direct counting derives T-duality in this subsector.

Now we consider the same problem after placing one D6-brane at nonzero FI parameter ( $B_{\mu \nu}$ background along 1234). Again, as in the previous paragraph, we work after Tdualizing along $x^{11}$, after which the extra D6-brane becomes a D5-brane along 012345 . The setting of section 3 was that D6 and N NS5-branes are placed at the same point of $\mathbb{R}^{3}$ in the 678 directions. Now T-dualizing along $x^{11}$, one finds a D5-brane on top of the $\mathbb{R}^{4} / \mathbb{Z}_{N}$ singularity of the unresolved Taub-NUT. Now, among the $N$ normalizable harmonic forms of $N$-centered Taub-NUT, $N-1$ of them are supported at the $Z_{N}$ singularity, where D5 is sitting. Since the fully winding D1-branes are forced to be bound to D5 at the tip due to the FI parameter, D1-branes stuck to D5 can still assume one of these $N-1$ bound state wavefunctions. The multi-particle index of the bounds is $P E\left[(N-1) I_{+} \frac{w^{\prime}}{1-w^{\prime}}\right]$. However, the last normalizable harmonic form of Taub-NUT is not localized at the tip, so D1-branes confined to D5 cannot be in this bound state. (The forbidden wavefunction is in the twisted Higgs branch.) Now, note that $n$ D1-branes can also form threshold bounds with single D5-brane, whose partition function is given by $P E\left[I_{-} \frac{w^{\prime}}{1-w^{\prime}}\right]$ [22]. (This extra contribution is also from the twisted Higgs branch, since the D2-D6 bounds still exist after displacing D6 away from NS5's.) This is an extra bound state caused by a D6-brane (at $\xi^{m} \neq 0$ ). Combining them all, one obtains

$$
P E\left[I_{-} \frac{w^{\prime}}{1-w^{\prime}}+(N-1) I_{+} \frac{w^{\prime}}{1-w^{\prime}}\right]=P E\left[N I_{+}\left(\epsilon_{1,2}, m\right) \frac{w^{\prime}}{1-w^{\prime}}\right] \cdot Z_{\text {extra }}\left(w^{\prime}\right)
$$

in the sector we are considering, where $Z_{\text {extra }}\left(w^{\prime}\right) \equiv \prod_{n=1}^{\infty} \frac{1}{1-\left(w^{\prime}\right)^{n}} \sim \eta\left(w^{\prime}\right)^{-1}$, and we used $I_{-}-I_{+}=1$ to get to the right hand side. So in this sector, one finds $\hat{Z}_{\text {string }}^{\text {IIA }}=Z_{\text {string }}^{\text {IIA }} Z_{\text {extra }}$. 
In fact, (4.5) is what one finds from the formulae of section 3 from our gauge theories. So extending this finding to the full BPS Hilbert space, we naturally conjecture $\hat{Z}_{\text {string }}^{\text {IIIA }}=$ $Z_{\text {string }}^{\text {IIA }} Z_{\text {extra }}$. Then the true partition function of the IIA little string theory is

$$
Z_{\mathrm{IIA}}\left(\alpha_{i}, \epsilon_{1,2}, m, q^{\prime}, w^{\prime}\right)=Z_{\mathrm{mom}}^{\mathrm{IIA}} \cdot \frac{\hat{Z}_{\mathrm{string}}^{\mathrm{IIA}}\left(\alpha_{i}, \epsilon_{1,2}, m, q^{\prime}, w^{\prime}\right)}{Z_{\mathrm{extra}}\left(w^{\prime}\right)}=\eta\left(w^{\prime}\right) Z_{\mathrm{mom}}^{\mathrm{IIA}} \hat{Z}_{\mathrm{string}}^{\mathrm{IIA}}
$$

which we shall use to check the T-duality relation (4.2). We checked (4.2) using (4.6) at $N=1,2,3$, as summarized in the following subsections. This will nontrivially support the T-duality of the strong-coupling little string spectra.

\subsection{One NS5-brane}

We start by considering the index of the U(1) IIB theory, although this should be a free theory. The perturbative contribution is given by

$$
Z_{\text {pert }}^{\mathrm{IIB}}\left(\epsilon_{ \pm}, m ; q\right)=P E\left[I_{+}\left(\epsilon_{ \pm}, m\right) \frac{q}{1-q}\right] .
$$

The $\mathrm{U}(1)$ instanton string partition function is given by

$$
Z_{\text {string }}^{\mathrm{IIB}}\left(\epsilon_{ \pm}, m ; q, w\right)=\sum_{k=0} w^{k} Z_{k}\left(\epsilon_{ \pm}, m ; q\right)
$$

where

$$
Z_{k}=\sum_{Y:|Y|=k} \prod_{s \in Y} \frac{\theta_{1}\left(q ; E(s)+m-\epsilon_{-}\right) \theta_{1}\left(q ; E(s)-m-\epsilon_{-}\right)}{\theta_{1}\left(q ; E(s)-\epsilon_{1}\right) \theta_{1}\left(q ; E(s)+\epsilon_{2}\right)},
$$

with

$$
E(s)=-\epsilon_{1} h(s)+\epsilon_{2} v(s) .
$$

The full index of the $\mathrm{U}(1)$ theory is given by

$$
Z_{\mathrm{IIB}}\left(\epsilon_{ \pm}, m ; q, w\right)=Z_{\text {pert }}^{\mathrm{IIB}}\left(\epsilon_{ \pm}, m ; q\right) Z_{\text {inst }}^{\mathrm{IIB}}\left(\epsilon_{ \pm}, m ; q, w\right)
$$

To further explain this index, consider the single instanton string index given by

$$
Z_{1}\left(\epsilon_{ \pm}, m ; q\right)=\frac{\theta_{1}\left(q ; m \pm \epsilon_{-}\right)}{\theta_{1}\left(q ; \epsilon_{1}\right) \theta_{1}\left(q ; \epsilon_{2}\right)} .
$$

where $\theta_{1}(q ; a \pm b) \equiv \theta_{1}(q ; a+b) \theta_{1}(q ; a-b)$. In terms of $Z_{1}$, we find up to high orders in $w$ and $q$ expansions that the multi-instanton string index is given by the Hecke transformation of $Z_{1}$,

$$
Z_{\text {inst }}\left(\epsilon_{ \pm}, m ; q, w\right)=\exp \left[\sum_{n=1}^{\infty} \frac{1}{n} w^{n} \sum_{\substack{a d=n \\ a, d \in \mathbb{Z}}} \sum_{b(\bmod d)} Z_{1}\left(a \epsilon_{ \pm}, a m ; \frac{a \tau+b}{d}\right)\right]
$$

where $q=e^{2 \pi i \tau}$. So we conjecture with confidence that this relation is exact. Its physical meaning is that, for $\mathrm{U}(1)$ instanton strings, the moduli space is given by a symmetric 
a)

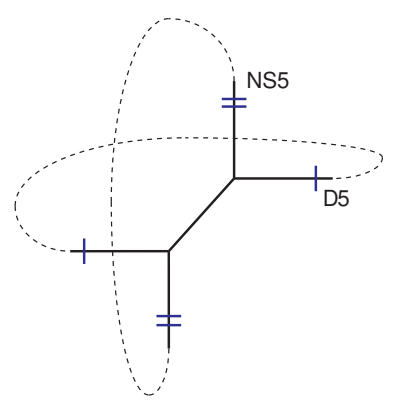

b)

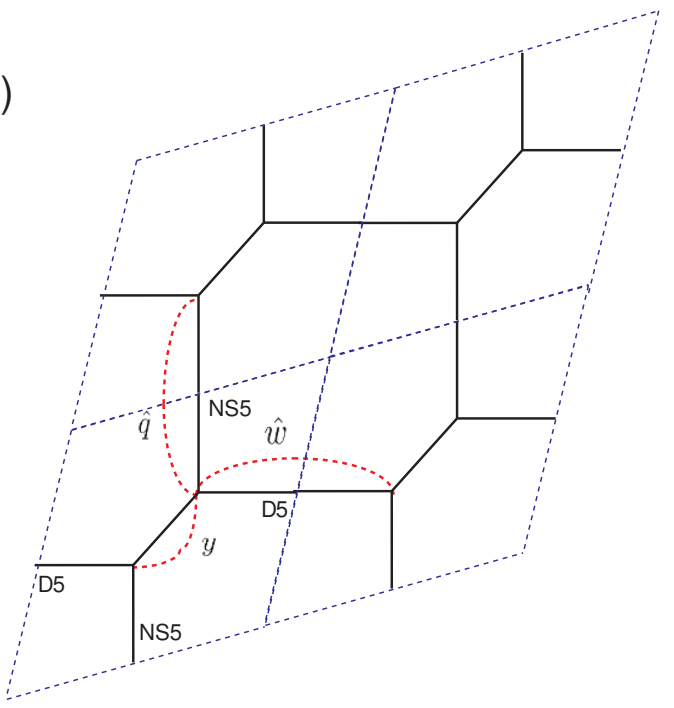

Figure 4. a) $(p, q)$ fivebranes web dual to rank 1 little string theory. b) Triality between three Kähler parameters, $\hat{q}=q y^{-1}, \hat{w}=w y^{-1}$, and $y$.

product of a single instanton moduli space, as elaborated in [6]. Since the Hecke transformation provides the partition function of the sigma model with symmetric product target space [29], our finding naturally supports the analysis of [6].

The partition function given by the Hecke transformation appears, for instance, in conformal field theories on symmetric product target spaces. This is closely related to the fact that the moduli-space of $\mathrm{U}(1)$ multi-instantons is a symmetric product of the single instanton moduli space $\mathbb{R}^{4}$. More precisely, the symmetric product CFT was suggested to be the theory at nonzero world-sheet theta angle $\theta=\pi[6,12]$. Since the elliptic genus would be insensitive to the continuous parameters, away from $\zeta^{I}=0, \theta=0$, it is natural to have (4.13).

On the IIA side, the $2 \mathrm{~d} \mathcal{N}=(0,4)$ quiver gauge theory itself has an enhanced $\mathcal{N}=$ $(4,4)$ SUSY, and becomes precisely the same to the $2 \mathrm{~d} \mathcal{N}=(4,4)$ ADHM gauge theory for IIB strings. Therefore,

$$
\hat{Z}_{\text {string }}^{\mathrm{IIA}}\left(\epsilon_{ \pm}, m ; q^{\prime}, w^{\prime}\right)=Z_{\text {inst }}^{\mathrm{IIB}}\left(\epsilon_{ \pm}, m, q^{\prime}, w^{\prime}\right) .
$$

The extra factor $Z_{\text {mom }}^{\mathrm{IIA}}$ on the IIA side is given by

$$
Z_{\mathrm{mom}}^{\mathrm{IIA}}\left(\epsilon_{ \pm}, m ; q^{\prime}\right)=P E\left[I_{-}\left(\epsilon_{ \pm}, m\right) \frac{q^{\prime}}{1-q^{\prime}}\right]=Z_{\mathrm{pert}}^{\mathrm{IIB}}\left(\epsilon_{ \pm}, m, q^{\prime}\right) Z_{\mathrm{extra}}\left(q^{\prime}\right)
$$

So the T-duality relation $(4.2)$ is equivalent to $\hat{Z}_{\mathrm{IIA}}\left(\epsilon_{ \pm}, m ; q^{\prime}, w^{\prime}\right) \equiv Z_{\text {mom }}^{\mathrm{IIA}} \hat{Z}_{\text {string }}^{\mathrm{IIA}}$ being invariant under the exchange of $q^{\prime}$ and $w^{\prime}$. The last property is in fact true, which can be understood as the geometric duality of the 5-brane web obtained by T-dualizing our IIA brane setting along $x^{5}[25]$, as shown in figure 4 . If we write $\hat{Z}_{\text {IIA }}\left(\epsilon_{ \pm}, m ; q^{\prime}, w^{\prime}\right)$ as

$$
\hat{Z}_{\mathrm{IIA}}\left(\epsilon_{ \pm}, m ; q^{\prime}, w^{\prime}\right)=P E\left[I_{-}\left(\epsilon_{ \pm}, m\right) z_{\mathrm{sp}}\left(\epsilon_{ \pm}, m, q^{\prime}, w^{\prime}\right)\right]
$$


$z_{\mathrm{sp}}\left(\epsilon_{ \pm}, m\right)$ is given by

$$
\begin{aligned}
& z_{\mathrm{sp}}\left(\epsilon_{ \pm}, m ; q^{\prime}, w^{\prime}\right)=\left(q^{\prime}+w^{\prime}\right)+\left(q^{\prime 2}+w^{\prime 2}\right)+\left(q^{\prime} w^{\prime}\right)\left[t u+\frac{t}{u}+\frac{1}{t u}+\frac{u}{t}-u y-\frac{y}{u}-\frac{u}{y}-\frac{1}{u y}\right] \\
& +q^{\prime 3}+w^{\prime 3}+\left(q^{\prime 2} w^{\prime}+q^{\prime} w^{\prime 2}\right)\left[t^{2} u^{2}+\frac{t^{2}}{u^{2}}+\frac{u^{2}}{t^{2}}+\frac{1}{t^{2} u^{2}}+t^{2}+\frac{1}{t^{2}}-t u^{2} y-\frac{t y}{u^{2}}-\frac{t u^{2}}{y}-\frac{t}{u^{2} y}\right. \\
& -\frac{y}{t u^{2}}-\frac{u^{2}}{t y}-\frac{1}{t u^{2} y}-\frac{u^{2} y}{t}+t u+\frac{t}{u}+\frac{1}{t u}+\frac{u}{t}-2 t y-\frac{2 t}{y}-\frac{2}{t y}-\frac{2 y}{t}+2 u^{2}+\frac{2}{u^{2}}-u y-\frac{y}{u} \\
& \left.-\frac{u}{y}-\frac{1}{u y}+y^{2}+\frac{1}{y^{2}}+4\right]+\left(q^{\prime 4}+w^{\prime 4}\right)+\left(q^{\prime 3} w^{\prime}+q^{\prime} w^{\prime 3}\right)\left[t^{3} u^{3}+\frac{t^{3}}{u^{3}}+\frac{u^{3}}{t^{3}}+\frac{1}{t^{3} u^{3}}+t^{3} u+\frac{t^{3}}{u}\right. \\
& +\frac{u}{t^{3}}+\frac{1}{t^{3} u}-t^{2} u^{3} y-\frac{t^{2} y}{u^{3}}-\frac{t^{2} u^{3}}{y}-\frac{t^{2}}{u^{3} y}-\frac{u^{3} y}{t^{2}}-\frac{y}{t^{2} u^{3}}-\frac{u^{3}}{t^{2} y}-\frac{1}{t^{2} u^{3} y}+t^{2} u^{2}+\frac{t^{2}}{u^{2}}+\frac{u^{2}}{t^{2}} \\
& +\frac{1}{t^{2} u^{2}}-2 t^{2} u y-\frac{2 t^{2} y}{u}-\frac{2 t^{2} u}{y}-\frac{2 t^{2}}{u y}-\frac{2 u y}{t^{2}}-\frac{2 y}{t^{2} u}-\frac{2 u}{t^{2} y}-\frac{2}{t^{2} u y}+2 t^{2}+\frac{2}{t^{2}}+2 t u^{3}+\frac{2 t}{u^{3}} \\
& +\frac{2}{t u^{3}}+\frac{2 u^{3}}{t}-2 t u^{2} y-\frac{2 t y}{u^{2}}-\frac{2 t u^{2}}{y}-\frac{2 t}{u^{2} y}-\frac{2 y}{t u^{2}}-\frac{2 u^{2}}{t y}-\frac{2}{t u^{2} y}-\frac{2 u^{2} y}{t}+t u y^{2}+\frac{t y^{2}}{u}+\frac{t u}{y^{2}} \\
& +\frac{t}{u y^{2}}+\frac{y^{2}}{t u}+\frac{u}{t y^{2}}+\frac{1}{t u y^{2}}+\frac{u y^{2}}{t}+6 t u+\frac{6 t}{u}+\frac{6}{t u}+\frac{6 u}{t}-4 t y-\frac{4 t}{y}-\frac{4}{t y}-\frac{4 y}{t}-u^{3} y-\frac{y}{u^{3}} \\
& \left.-\frac{u^{3}}{y}-\frac{1}{u^{3} y}+u^{2} y^{2}+\frac{y^{2}}{u^{2}}+\frac{u^{2}}{y^{2}}+\frac{1}{u^{2} y^{2}}+4 u^{2}+\frac{4}{u^{2}}-5 u y-\frac{5 y}{u}-\frac{5 u}{y}-\frac{5}{u y}+2 y^{2}+\frac{2}{y^{2}}+8\right] \\
& +\left(q^{\prime 2} w^{\prime 2}\right)\left[u^{4} t^{4}+u^{2} t^{4}+\frac{t^{4}}{u^{2}}+\frac{t^{4}}{u^{4}}+t^{4}+u^{3} t^{3}+2 u t^{3}-u^{4} y t^{3}-2 u^{2} y t^{3}-2 y t^{3}+\frac{2 t^{3}}{u}-\frac{2 y t^{3}}{u^{2}}\right. \\
& +\frac{t^{3}}{u^{3}}-\frac{y t^{3}}{u^{4}}-\frac{u^{4} t^{3}}{y}-\frac{2 u^{2} t^{3}}{y}-\frac{2 t^{3}}{y}-\frac{2 t^{3}}{u^{2} y}-\frac{t^{3}}{u^{4} y}+2 u^{4} t^{2}+7 u^{2} t^{2}+u^{2} y^{2} t^{2}+\frac{y^{2} t^{2}}{u^{2}}+y^{2} t^{2} \\
& -2 u^{3} y t^{2}-5 u y t^{2}-\frac{5 y t^{2}}{u}+\frac{7 t^{2}}{u^{2}}-\frac{2 y t^{2}}{u^{3}}+\frac{2 t^{2}}{u^{4}}-\frac{2 u^{3} t^{2}}{y}-\frac{5 u t^{2}}{y}-\frac{5 t^{2}}{u y}-\frac{2 t^{2}}{u^{3} y}+\frac{u^{2} t^{2}}{y^{2}}+\frac{t^{2}}{y^{2}} \\
& +\frac{t^{2}}{u^{2} y^{2}}+9 t^{2}+5 u^{3} t+u^{3} y^{2} t+4 u y^{2} t+\frac{4 y^{2} t}{u}+\frac{y^{2} t}{u^{3}}+15 u t-u^{4} y t-7 u^{2} y t-12 y t+\frac{15 t}{u} \\
& -\frac{7 y t}{u^{2}}+\frac{5 t}{u^{3}}-\frac{y t}{u^{4}}-\frac{u^{4} t}{y}-\frac{7 u^{2} t}{y}-\frac{12 t}{y}-\frac{7 t}{u^{2} y}-\frac{t}{u^{4} y}+\frac{u^{3} t}{y^{2}}+\frac{4 u t}{y^{2}}+\frac{4 t}{u y^{2}}+\frac{t}{u^{3} y^{2}}+\frac{2 u^{4}}{t^{2}} \\
& +\frac{u^{4}}{t^{4}}+2 u^{4}+\frac{u^{3}}{t^{3}}-u y^{3}+\frac{7 u^{2}}{t^{2}}+\frac{u^{2}}{t^{4}}+12 u^{2}+\frac{u^{2} y^{2}}{t^{2}}+2 u^{2} y^{2}+\frac{y^{2}}{t^{2}}+\frac{4 y^{2}}{t u}+\frac{2 y^{2}}{u^{2}}+\frac{y^{2}}{t^{2} u^{2}}+\frac{y^{2}}{t u^{3}} \\
& +5 y^{2}+\frac{2 u}{t^{3}}-4 u^{3} y-14 u y-\frac{2 u^{3} y}{t^{2}}-\frac{5 u y}{t^{2}}+\frac{9}{t^{2}}-\frac{u^{4} y}{t^{3}}-\frac{2 u^{2} y}{t^{3}}-\frac{2 y}{t^{3}}+\frac{1}{t^{4}}-\frac{y^{3}}{u}-\frac{14 y}{u}+\frac{15}{t u} \\
& -\frac{5 y}{t^{2} u}+\frac{2}{t^{3} u}+\frac{12}{u^{2}}-\frac{7 y}{t u^{2}}+\frac{7}{t^{2} u^{2}}-\frac{2 y}{t^{3} u^{2}}+\frac{1}{t^{4} u^{2}}-\frac{4 y}{u^{3}}+\frac{5}{t u^{3}}-\frac{2 y}{t^{2} u^{3}}+\frac{1}{t^{3} u^{3}}+\frac{2}{u^{4}}-\frac{y}{t u^{4}}+\frac{2}{t^{2} u^{4}} \\
& -\frac{y}{t^{3} u^{4}}+\frac{1}{t^{4} u^{4}}-\frac{4 u^{3}}{y}-\frac{14 u}{y}-\frac{u^{4}}{t y}-\frac{7 u^{2}}{t y}-\frac{12}{t y}-\frac{2 u^{3}}{t^{2} y}-\frac{5 u}{t^{2} y}-\frac{u^{4}}{t^{3} y}-\frac{2 u^{2}}{t^{3} y}-\frac{2}{t^{3} y}-\frac{14}{u y}-\frac{5}{t^{2} u y} \\
& -\frac{7}{t u^{2} y}-\frac{2}{t^{3} u^{2} y}-\frac{4}{u^{3} y}-\frac{2}{t^{2} u^{3} y}-\frac{1}{t u^{4} y}-\frac{1}{t^{3} u^{4} y}+\frac{2 u^{2}}{y^{2}}+\frac{5}{y^{2}}+\frac{u^{3}}{t y^{2}}+\frac{4 u}{t y^{2}}+\frac{u^{2}}{t^{2} y^{2}}+\frac{1}{t^{2} y^{2}}+\frac{4}{t u y^{2}} \\
& +\frac{2}{u^{2} y^{2}}+\frac{1}{t^{2} u^{2} y^{2}}+\frac{1}{t u^{3} y^{2}}-\frac{u}{y^{3}}-\frac{1}{u y^{3}}+22+\frac{5 u^{3}}{t}+\frac{u^{3} y^{2}}{t}+\frac{4 u y^{2}}{t}+\frac{15 u}{t}-\frac{u^{4} y}{t}-\frac{7 u^{2} y}{t} \\
& \left.-\frac{12 y}{t}\right]+\cdots \text {. }
\end{aligned}
$$

where $t=e^{2 \pi i \epsilon_{+}}, u=e^{2 \pi i \epsilon_{-}}, y=e^{2 \pi i m}$. We checked the symmetry of $q^{\prime} \leftrightarrow w^{\prime}$ exchange up to 5 th orders in $q^{\prime}$ and $w^{\prime}$. 
Furthermore, defining the following variables,

$$
\hat{q}=q y^{-1}, \quad \hat{w}=w y^{-1} .
$$

triality of exchanging $(\hat{q}, \hat{w}, y)$ has been discovered in [25]. This is also a geometric duality of figure 4. Triality is simply realized on the universal covering of the torus, as a subgroup of $\operatorname{Sp}(4, Z)$ duality. To deal with $(\hat{q}, \hat{w}, y)$ in equal footing, we redefine the index, including extra perturbative contributions at $y \ll 1$, as

$$
\tilde{Z}\left(\epsilon_{ \pm} ; \hat{q}, \hat{w}, y\right)=P E\left[I_{\mathrm{com}}\left(\epsilon_{ \pm}\right) y\right] \hat{Z}_{\mathrm{IIA}}
$$

$I_{\mathrm{com}}\left(\epsilon_{ \pm}\right)$is given by

$$
I_{\mathrm{com}}\left(\epsilon_{ \pm}\right)=\frac{1}{2 \sinh \frac{2 \pi i \epsilon_{1}}{2} 2 \sinh \frac{2 \pi i \epsilon_{2}}{2}}=\frac{t}{(1-t u)\left(1-t u^{-1}\right)} .
$$

Writing $\tilde{Z}$ as

$$
\tilde{Z}\left(\epsilon_{ \pm} ; \hat{q}, \hat{w}, y\right)=P E\left[I_{\mathrm{com}} \tilde{z}_{\mathrm{sp}}\left(\epsilon_{ \pm} ; \hat{q}, \hat{w}, y\right)\right]
$$

$\tilde{z}_{\mathrm{sp}}$ is given by

$$
\begin{aligned}
& \hat{z}_{s p}\left(\epsilon_{ \pm} ; \hat{q}, \hat{w}, y\right)=\hat{q}+\hat{w}+y-\left(u+u^{-1}\right)(\hat{q} \hat{w}+\hat{q} y+\hat{w} y)+\frac{\left(1+u^{2}\right)\left(t+u+t^{2} u+t u^{2}\right)}{t u^{2}} \hat{q} \hat{w} y \\
& +\left(\hat{q}^{2} \hat{w}+\hat{q} \hat{w}^{2}+\hat{q}^{2} y+\hat{q} y^{2}+\hat{w}^{2} y+\hat{w} y^{2}\right)-\left(u+u^{-1}\right)\left(\hat{q}^{2} \hat{w}^{2}+\hat{q}^{2} y^{2}+\hat{w}^{2} y^{2}\right) \\
& -\frac{\left(u^{2}+1\right)\left(t^{2}\left(u^{2}+1\right)+2 t u+u^{2}+1\right)}{t u^{2}} \hat{q} \hat{w} y(\hat{q}+\hat{w}+y) \\
& +\left(\hat{q}^{3} \hat{w}^{2}+\hat{q}^{2} \hat{w}^{3}+\hat{q}^{3} y^{2}+\hat{q}^{2} y^{3}+\hat{w}^{3} y^{2}+\hat{w}^{2} y^{3}\right)+\frac{\left(1+u^{2}\right)\left(t+u+t^{2} u+t u^{2}\right)}{t u^{2}} \hat{q} \hat{w} y\left(\hat{q}^{2}+\hat{w}^{2}+y^{2}\right) \\
& +\frac{t^{4}\left(u^{5}+u^{3}+u\right)+t^{3}\left(u^{6}+4 u^{4}+4 u^{2}+1\right)}{t^{2} u^{3}} \hat{q} \hat{w} y(\hat{q} \hat{w}+\hat{q} y+\hat{w} y) \\
& +\frac{t^{2}\left(3 u^{4}+7 u^{2}+3\right) u+t\left(u^{6}+4 u^{4}+4 u^{2}+1\right)+u^{5}+u^{3}+u}{t^{2} u^{3}} \hat{q} \hat{w} y(\hat{q} \hat{w}+\hat{q} y+\hat{w} y) \\
& -\left(u+u^{-1}\right)\left(\hat{q}^{3} \hat{w}^{3}+\hat{q}^{3} y^{3}+\hat{w}^{3} y^{3}\right) \\
& -\frac{\left(u^{2}+1\right)\left(t^{4}\left(u^{4}+u^{2}+1\right)+3 t^{3}\left(u^{3}+u\right)\right)}{t^{2} u^{3}} \hat{q} \hat{w} y\left(\hat{q}^{2} \hat{w}+\hat{q} \hat{w}^{2}+\hat{q}^{2} y+\hat{q} y^{2}+\hat{w}^{2} y+\hat{w} y^{2}\right) \\
& -\frac{\left(u^{2}+1\right)\left(2 t^{2}\left(u^{4}+3 u^{2}+1\right)+3 t\left(u^{3}+u\right)+u^{4}+u^{2}+1\right)}{t^{2} u^{3}} \hat{w} y\left(\hat{q}^{2} \hat{w}+\hat{q} \hat{w}^{2}+(\mathrm{cyclic})\right) \\
& +\cdots
\end{aligned}
$$

reconfirming the expected triality of [25]. It is curious to note that the triality implies the T-duality of IIA/IIB strings.

\subsection{Two NS5-branes}

The index of $\mathrm{U}(2)$ IIB little string theory is given by

$$
Z_{\mathrm{IIB}}\left(\alpha_{i}, \epsilon_{ \pm}, m ; q, w\right)=Z_{\mathrm{pert}}^{\mathrm{IIB}}\left(\alpha_{i}, \epsilon_{ \pm}, m ; q\right) Z_{\mathrm{inst}}^{\mathrm{IIB}}\left(\alpha_{i}, \epsilon_{ \pm}, m ; q, w\right),
$$


where

$$
Z_{\mathrm{pert}}^{\mathrm{IIB}}\left(\alpha_{i}, \epsilon_{ \pm}, m ; q\right)=P E\left[I_{+} v_{1}+\left(2 I_{+}+I_{+}\left(v_{1}+v_{1}^{-1}\right)\right) \frac{q}{1-q}\right]=P E\left[I_{+} \frac{v_{1}+v_{2}+2 v_{1} v_{2}}{1-v_{1} v_{2}}\right]
$$

with $v_{1}=e^{2 \pi i \alpha_{12}}$, and $v_{2} \equiv q v_{1}^{-1} . I_{+}$is given by eq. (2.12). $Z_{\text {inst }}^{\mathrm{IIB}}$ is given by

$$
Z_{\text {inst }}^{\mathrm{IIB}}\left(\alpha_{i}, \epsilon_{ \pm}, m ; q, w\right)=\sum_{k=0}^{\infty} w^{k} Z_{k}\left(\alpha_{i}, \epsilon_{ \pm}, m ; q\right) .
$$

$Z_{k}$ is obtained from eq. (2.7). Expanding $Z_{\text {inst }}^{\mathrm{IIB}}\left(\alpha_{i}, \epsilon_{ \pm}, m ; q, w\right)$ with $w, v_{1}, v_{2}=q v_{1}^{-1}$, one obtains

$$
\begin{aligned}
& Z_{\text {inst }}^{\mathrm{IIB}}\left(\epsilon_{ \pm}, m ; w, v_{i}\right) \\
&= 1-w \frac{2 t(u-y)(u y-1)}{y(t-u)(t u-1)}+w\left(v_{1}+v_{2}\right) \frac{\left(t^{2}+1\right)(t-y)(t y-1)(y-u)(u y-1)}{t y^{2}(t-u)(t u-1)} \\
&+w\left(v_{1}^{2}+v_{2}^{2}\right) \frac{\left(t^{2}+1\right)\left(t^{4}+1\right)(t-y)(t y-1)(y-u)(u y-1)}{t^{3} y^{2}(t-u)(t u-1)} \\
&+w v_{1} v_{2} \frac{2(t-y)(t y-1)(y-u)(u y-1)\left(t^{2} u y+t(u-y)(u y-1)+u y\right)}{t u y^{3}(t-u)(t u-1)} \\
&+w\left(v_{1}^{2} v_{2}+v_{1} v_{2}^{2}\right) \frac{\left(t^{2}+1\right)(t-y)(t y-1)(y-u)(u y-1)}{t^{3} u y^{3}(t-u)(t u-1)} \\
& \times\left\{-t(t+u)(1+t u)\left(1+y^{2}\right)+\left(t+u+t^{2} u\right)(1+t(t+u)) y\right\}+\cdots
\end{aligned}
$$

The index for the rank 2 IIA little string theory is given by

$$
Z_{\mathrm{IIA}}=Z_{\mathrm{extra}}(q)^{-1} Z_{\mathrm{mom}}^{\mathrm{IIA}}\left(\epsilon_{ \pm}, m ; w\right) \hat{Z}_{\text {string }}^{\mathrm{IIA}}\left(\alpha_{i}, \epsilon_{ \pm}, m ; w, q\right)
$$

where we inserted $q^{\prime}=w, w^{\prime}=q . Z_{\text {extra }}(q)$ is given by

$$
Z_{\text {extra }}(q)=P E\left[\frac{q}{1-q}\right]=P E\left[\frac{v_{1} v_{2}}{1-v_{1} v_{2}}\right] .
$$

$Z_{\text {mom }}^{\mathrm{IIA}}\left(\epsilon_{ \pm}, m ; w\right)$ is given by

$$
Z_{\mathrm{N}=2 \operatorname{mom}}^{\mathrm{IIA}}\left(\epsilon_{ \pm}, m ; w\right)=P E\left[2 I_{-}\left(\epsilon_{ \pm}, m\right) \frac{w}{1-w}\right] .
$$

$\hat{Z}_{\text {string }}^{\text {IIA }}\left(\alpha_{i}, \epsilon_{ \pm}, m ; w, q\right)$ takes the form of

$$
\hat{Z}_{\text {string }}^{\text {IIA }}\left(\alpha_{i}, \epsilon_{ \pm}, m ; w, q\right)=\sum_{n_{1}, n_{2}=0}^{\infty}\left(v_{1}\right)^{n_{1}}\left(v_{2}\right)^{n_{2}} Z_{\text {string }}^{\left(n_{1}, n_{2}\right)}\left(\epsilon_{ \pm}, m ; w\right),
$$

Note that $Z_{\text {string }}^{\left(n_{1}, n_{2}\right)}\left(\epsilon_{ \pm}, m ; w\right)=Z_{\text {string }}^{\left(n_{2}, n_{1}\right)}\left(\epsilon_{ \pm}, m ; w\right)$, from the symmetry of the quiver. $Z_{\text {string }}^{\left(n_{1}, n_{2}\right)}\left(\epsilon_{ \pm}, m ; w\right)$ can be easily obtained from (3.15). For instance,

$$
Z_{\text {string }}^{(1,0)}\left(\epsilon_{ \pm}, m ; w\right)=\frac{\theta_{1}\left(w, m \pm \epsilon_{+}\right)}{\theta_{1}\left(w, \epsilon_{1}\right) \theta_{1}\left(w, \epsilon_{2}\right)}, \quad Z_{\text {string }}^{(1,1)}\left(\epsilon_{ \pm}, m ; w\right)=\frac{\theta_{1}\left(w, m \pm \epsilon_{-}\right)^{2}}{\theta_{1}\left(w, \epsilon_{1}\right)^{2} \theta_{1}\left(w, \epsilon_{2}\right)^{2}}
$$




$$
Z_{\text {string }}^{(2,0)}\left(\epsilon_{ \pm}, m ; w\right)=Z_{\text {string }}^{(1,0)} \cdot\left(\frac{\theta_{1}\left(w ; \epsilon_{+}+\epsilon_{1} \pm m\right)}{\theta_{1}\left(w ; 2 \epsilon_{1}\right) \theta_{1}\left(w ; \epsilon_{1}-\epsilon_{2}\right)}-\left(\epsilon_{1} \leftrightarrow \epsilon_{2}\right)\right)
$$

We write the indices of the IIA/IIB little string theories as

$$
\begin{aligned}
& Z_{\mathrm{IIB}}\left(\alpha_{i}, \epsilon_{ \pm}, m ; w, v_{i}\right)=P E\left[I_{\mathrm{com}}(t, u) \sum_{i, j, k=0}^{\infty} F_{i j k}^{\mathrm{IIB}}(t, u, y) w^{i} v_{1}^{j} v_{2}^{k}\right], \\
& Z_{\mathrm{IIA}}\left(\alpha_{i}, \epsilon_{ \pm}, m ; w, v_{i}\right)=P E\left[I_{\mathrm{com}}(t, u) \sum_{i, j, k=0}^{\infty} F_{i j k}^{\mathrm{IIA}}(t, u, y) w^{i} v_{1}^{j} v_{2}^{k}\right],
\end{aligned}
$$

where $I_{\text {com }}$ is given by eq. (4.20). The coefficients $F_{i j k}^{\mathrm{IIB}}(t, u, y)$ are polynomials of $t=e^{2 \pi i \epsilon_{+}}$, $u=e^{2 \pi i \epsilon_{-}}$, and $y=e^{2 \pi i m}$. It is easily checked that $F_{i j k}^{\mathrm{IIB}}(t, u, y)=F_{i k j}^{\mathrm{IIB}}(t, u, y)$.

T-duality implies that $F_{i j k}^{\mathrm{IIA}}=F_{i j k}^{\mathrm{IIB}} \equiv F_{i j k}$. We check T-daulity by comparing $F_{i j k}^{\mathrm{IIA}}$ and $F_{i j k}^{\mathrm{IIB}}$. We checked the agreements for

$$
\begin{aligned}
& F_{000}=1, \quad F_{010}=-t-\frac{1}{t}+y+\frac{1}{y}, \quad F_{011}=-2 t-\frac{2}{t}+2 y+\frac{2}{y} \\
& F_{020}=0, \quad F_{021}=-t-\frac{1}{t}+y+\frac{1}{y}, \quad F_{022}=-2 t-\frac{2}{t}+2 y+\frac{2}{y} \\
& F_{100}=-2 u-\frac{2}{u}+2 y+\frac{2}{y}, \\
& F_{110}=-t^{2} u-\frac{t^{2}}{u}-\frac{u}{t^{2}}-\frac{1}{t^{2} u}+t^{2} y+\frac{t^{2}}{y}+\frac{y}{t^{2}}+\frac{1}{t^{2} y}+t u y+\frac{t y}{u}+\frac{t u}{y}+\frac{t}{u y}+\frac{y}{t u}+\frac{u}{t y} \\
& +\frac{1}{t u y}+\frac{u y}{t}-t y^{2}-\frac{t}{y^{2}}-\frac{1}{t y^{2}}-\frac{y^{2}}{t}-2 t-\frac{2}{t}-2 u-\frac{2}{u}+2 y+\frac{2}{y} \\
& F_{111}=-2 t^{2} u-\frac{2 u}{t^{2}}-\frac{2 t^{2}}{u}-\frac{2}{t^{2} u}+2 t^{2} y+\frac{2 y}{t^{2}}+\frac{2}{t^{2} y}+\frac{2 t^{2}}{y}-2 t u^{2}-\frac{2 u^{2}}{t}-\frac{2 t}{u^{2}}-\frac{2}{t u^{2}}+6 t u y \\
& +\frac{6 u y}{t}+\frac{6 t y}{u}+\frac{6 y}{t u}+\frac{6 u}{t y}+\frac{6 t}{u y}+\frac{6}{t u y}+\frac{6 t u}{y}-4 t y^{2}-\frac{4 y^{2}}{t}-\frac{4 t}{y^{2}}-\frac{4}{t y^{2}}-12 t-\frac{12}{t}+2 u^{2} y \\
& +\frac{2 y}{u^{2}}+\frac{2}{u^{2} y}+\frac{2 u^{2}}{y}-4 u y^{2}-\frac{4 y^{2}}{u}-\frac{4 u}{y^{2}}-\frac{4}{u y^{2}}-12 u-\frac{12}{u}+2 y^{3}+\frac{2}{y^{3}}+14 y+\frac{14}{y} \\
& F_{120}=-t^{4} u-\frac{t^{4}}{u}-\frac{u}{t^{4}}-\frac{1}{t^{4} u}+t^{4} y+\frac{t^{4}}{y}+\frac{y}{t^{4}}+\frac{1}{t^{4} y}+t^{3} u y+\frac{t^{3} y}{u}+\frac{t^{3} u}{y}+\frac{t^{3}}{u y}+\frac{u y}{t^{3}}+\frac{y}{t^{3} u} \\
& +\frac{u}{t^{3} y}+\frac{1}{t^{3} u y}-t^{3} y^{2}-\frac{t^{3}}{y^{2}}-\frac{y^{2}}{t^{3}}-\frac{1}{t^{3} y^{2}}-2 t^{3}-\frac{2}{t^{3}}-2 t^{2} u-\frac{2 t^{2}}{u}-\frac{2 u}{t^{2}}-\frac{2}{t^{2} u}+2 t^{2} y+\frac{2 t^{2}}{y} \\
& +\frac{2 y}{t^{2}}+\frac{2}{t^{2} y}+t u y+\frac{t y}{u}+\frac{t u}{y}+\frac{t}{u y}+\frac{y}{t u}+\frac{u}{t y}+\frac{1}{t u y}+\frac{u y}{t}-t y^{2}-\frac{t}{y^{2}}-\frac{1}{t y^{2}}-\frac{y^{2}}{t}-2 t-\frac{2}{t} \\
& -2 u-\frac{2}{u}+2 y+\frac{2}{y} \\
& F_{121}=-t^{4} u+y t^{4}-\frac{t^{4}}{u}+\frac{t^{4}}{y}-u^{2} t^{3}-2 y^{2} t^{3}+3 u y t^{3}+\frac{3 y t^{3}}{u}-\frac{t^{3}}{u^{2}}+\frac{3 u t^{3}}{y}+\frac{3 t^{3}}{u y}-\frac{2 t^{3}}{y^{2}}-6 t^{3} \\
& +y^{3} t^{2}-3 u y^{2} t^{2}-11 u t^{2}+2 u^{2} y t^{2}+\frac{2 y t^{2}}{u^{2}}+12 y t^{2}-\frac{3 y^{2} t^{2}}{u}-\frac{11 t^{2}}{u}+\frac{2 u^{2} t^{2}}{y}+\frac{12 t^{2}}{y}+\frac{2 t^{2}}{u^{2} y} \\
& -\frac{3 u t^{2}}{y^{2}}-\frac{3 t^{2}}{u y^{2}}+\frac{t^{2}}{y^{3}}+u y^{3} t+\frac{y^{3} t}{u}-5 u^{2} t-u^{2} y^{2} t-9 y^{2} t+13 u y t+\frac{13 y t}{u}-\frac{y^{2} t}{u^{2}}-\frac{5 t}{u^{2}}+\frac{13 u t}{y} \\
& +\frac{13 t}{u y}-\frac{u^{2} t}{y^{2}}-\frac{9 t}{y^{2}}-\frac{t}{u^{2} y^{2}}+\frac{u t}{y^{3}}+\frac{t}{u y^{3}}-24 t+\frac{y^{3}}{t^{2}}+\frac{y^{3}}{t u}+2 y^{3}-6 u y^{2}-20 u+\frac{2 u^{2} y}{t^{2}}+4 u^{2} y \\
& +\frac{3 u y}{t^{3}}+\frac{12 y}{t^{2}}+\frac{y}{t^{4}}+\frac{13 y}{t u}+\frac{3 y}{t^{3} u}+\frac{4 y}{u^{2}}+\frac{2 y}{t^{2} u^{2}}+22 y-\frac{3 u y^{2}}{t^{2}}-\frac{11 u}{t^{2}}-\frac{u^{2}}{t^{3}}-\frac{2 y^{2}}{t^{3}}-\frac{6}{t^{3}}-\frac{u}{t^{4}}
\end{aligned}
$$




$$
\begin{aligned}
& -\frac{6 y^{2}}{u}-\frac{20}{u}-\frac{3 y^{2}}{t^{2} u}-\frac{11}{t^{2} u}-\frac{1}{t^{4} u}-\frac{y^{2}}{t u^{2}}-\frac{5}{t u^{2}}-\frac{1}{t^{3} u^{2}}+\frac{4 u^{2}}{y}+\frac{22}{y}+\frac{13 u}{t y}+\frac{2 u^{2}}{t^{2} y}+\frac{12}{t^{2} y}+\frac{3 u}{t^{3} y} \\
& +\frac{1}{t^{4} y}+\frac{13}{t u y}+\frac{3}{t^{3} u y}+\frac{4}{u^{2} y}+\frac{2}{t^{2} u^{2} y}-\frac{6 u}{y^{2}}-\frac{u^{2}}{t y^{2}}-\frac{9}{t y^{2}}-\frac{3 u}{t^{2} y^{2}}-\frac{2}{t^{3} y^{2}}-\frac{6}{u y^{2}}-\frac{3}{t^{2} u y^{2}}-\frac{1}{t u^{2} y^{2}} \\
& +\frac{2}{y^{3}}+\frac{u}{t y^{3}}+\frac{1}{t^{2} y^{3}}+\frac{1}{t u y^{3}}+\frac{u y^{3}}{t}-\frac{5 u^{2}}{t}-\frac{u^{2} y^{2}}{t}-\frac{9 y^{2}}{t}+\frac{13 u y}{t}-\frac{24}{t} \\
& F_{122}=-2 u t^{4}+2 y t^{4}-\frac{2 t^{4}}{u}+\frac{2 t^{4}}{y}-2 u^{2} t^{3}-4 y^{2} t^{3}+6 u y t^{3}+\frac{6 y t^{3}}{u}-\frac{2 t^{3}}{u^{2}}+\frac{6 u t^{3}}{y}+\frac{6 t^{3}}{u y}-\frac{4 t^{3}}{y^{2}} \\
& -12 t^{3}-2 u^{3} t^{2}+2 y^{3} t^{2}-8 u y^{2} t^{2}-32 u t^{2}+8 u^{2} y t^{2}+\frac{8 y t^{2}}{u^{2}}+32 y t^{2}-\frac{8 y^{2} t^{2}}{u}-\frac{32 t^{2}}{u}-\frac{2 t^{2}}{u^{3}}+\frac{8 u^{2} t^{2}}{y} \\
& +\frac{32 t^{2}}{y}+\frac{8 t^{2}}{u^{2} y}-\frac{8 u t^{2}}{y^{2}}-\frac{8 t^{2}}{u y^{2}}+\frac{2 t^{2}}{y^{3}}+6 u y^{3} t+\frac{6 y^{3} t}{u}-24 u^{2} t-8 u^{2} y^{2} t-36 y^{2} t+2 u^{3} y t+52 u y t \\
& +\frac{52 y t}{u}+\frac{2 y t}{u^{3}}-\frac{8 y^{2} t}{u^{2}}-\frac{24 t}{u^{2}}+\frac{2 u^{3} t}{y}+\frac{52 u t}{y}+\frac{52 t}{u y}+\frac{2 t}{u^{3} y}-\frac{8 u^{2} t}{y^{2}}-\frac{36 t}{y^{2}}-\frac{8 t}{u^{2} y^{2}}+\frac{6 u t}{y^{3}}+\frac{6 t}{u y^{3}} \\
& -88 t-2 u y^{4}-4 u^{3}+2 u^{2} y^{3}+\frac{2 y^{3}}{t^{2}}+\frac{6 y^{3}}{t u}+\frac{2 y^{3}}{u^{2}}+14 y^{3}-32 u y^{2}-88 u+\frac{8 u^{2} y}{t^{2}}+24 u^{2} y+\frac{6 u y}{t^{3}} \\
& +\frac{32 y}{t^{2}}+\frac{2 y}{t^{4}}+\frac{52 y}{t u}+\frac{6 y}{t^{3} u}+\frac{24 y}{u^{2}}+\frac{8 y}{t^{2} u^{2}}+\frac{2 y}{t u^{3}}+94 y-\frac{2 u^{3}}{t^{2}}-\frac{8 u y^{2}}{t^{2}}-\frac{32 u}{t^{2}}-\frac{2 u^{2}}{t^{3}}-\frac{4 y^{2}}{t^{3}}-\frac{12}{t^{3}} \\
& -\frac{2 u}{t^{4}}-\frac{2 y^{4}}{u}-\frac{32 y^{2}}{u}-\frac{88}{u}-\frac{8 y^{2}}{t^{2} u}-\frac{32}{t^{2} u}-\frac{2}{t^{4} u}-\frac{8 y^{2}}{t u^{2}}-\frac{24}{t u^{2}}-\frac{2}{t^{3} u^{2}}-\frac{4}{u^{3}}-\frac{2}{t^{2} u^{3}}+\frac{24 u^{2}}{y}+\frac{94}{y} \\
& +\frac{2 u^{3}}{t y}+\frac{52 u}{t y}+\frac{8 u^{2}}{t^{2} y}+\frac{32}{t^{2} y}+\frac{6 u}{t^{3} y}+\frac{2}{t^{4} y}+\frac{52}{t u y}+\frac{6}{t^{3} u y}+\frac{24}{u^{2} y}+\frac{8}{t^{2} u^{2} y}+\frac{2}{t u^{3} y}-\frac{32 u}{y^{2}}-\frac{8 u^{2}}{t y^{2}} \\
& -\frac{36}{t y^{2}}-\frac{8 u}{t^{2} y^{2}}-\frac{4}{t^{3} y^{2}}-\frac{32}{u y^{2}}-\frac{8}{t^{2} u y^{2}}-\frac{8}{t u^{2} y^{2}}+\frac{2 u^{2}}{y^{3}}+\frac{14}{y^{3}}+\frac{6 u}{t y^{3}}+\frac{2}{t^{2} y^{3}}+\frac{6}{t u y^{3}}+\frac{2}{u^{2} y^{3}}-\frac{2 u}{y^{4}} \\
& -\frac{2}{u y^{4}}+\frac{6 u y^{3}}{t}-\frac{24 u^{2}}{t}-\frac{8 u^{2} y^{2}}{t}-\frac{36 y^{2}}{t}+\frac{2 u^{3} y}{t}+\frac{52 u y}{t}-\frac{88}{t} \\
& F_{200}=-2 u-\frac{2}{u}+2 y+\frac{2}{y}, \\
& F_{210}=-t^{3} u^{2}-\frac{t^{3}}{u^{2}}-\frac{u^{2}}{t^{3}}-\frac{1}{t^{3} u^{2}}+t^{3} u y+\frac{t^{3} y}{u}+\frac{t^{3} u}{y}+\frac{t^{3}}{u y}+\frac{u y}{t^{3}}+\frac{y}{t^{3} u}+\frac{u}{t^{3} y}+\frac{1}{t^{3} u y}-2 t^{3} \\
& -\frac{2}{t^{3}}+t^{2} u^{2} y+\frac{t^{2} y}{u^{2}}+\frac{t^{2} u^{2}}{y}+\frac{t^{2}}{u^{2} y}+\frac{u^{2} y}{t^{2}}+\frac{y}{t^{2} u^{2}}+\frac{u^{2}}{t^{2} y}+\frac{1}{t^{2} u^{2} y}-t^{2} u y^{2}-\frac{t^{2} y^{2}}{u}-\frac{t^{2} u}{y^{2}}-\frac{t^{2}}{u y^{2}} \\
& -\frac{u y^{2}}{t^{2}}-\frac{y^{2}}{t^{2} u}-\frac{u}{t^{2} y^{2}}-\frac{1}{t^{2} u y^{2}}-3 t^{2} u-\frac{3 t^{2}}{u}-\frac{3 u}{t^{2}}-\frac{3}{t^{2} u}+3 t^{2} y+\frac{3 t^{2}}{y}+\frac{3 y}{t^{2}}+\frac{3}{t^{2} y}-2 t u^{2}-\frac{2 t}{u^{2}} \\
& -\frac{2}{t u^{2}}-\frac{2 u^{2}}{t}+4 t u y+\frac{4 t y}{u}+\frac{4 t u}{y}+\frac{4 t}{u y}+\frac{4 y}{t u}+\frac{4 u}{t y}+\frac{4}{t u y}+\frac{4 u y}{t}-2 t y^{2}-\frac{2 t}{y^{2}}-\frac{2}{t y^{2}}-\frac{2 y^{2}}{t}-8 t \\
& -\frac{8}{t}+u^{2} y+\frac{y}{u^{2}}+\frac{u^{2}}{y}+\frac{1}{u^{2} y}-2 u y^{2}-\frac{2 y^{2}}{u}-\frac{2 u}{y^{2}}-\frac{2}{u y^{2}}-6 u-\frac{6}{u}+y^{3}+\frac{1}{y^{3}}+7 y+\frac{7}{y} \\
& F_{211}=-t y^{4}-u y^{4}-\frac{y^{4}}{t}-\frac{y^{4}}{u}+2 t^{2} y^{3}+2 u^{2} y^{3}+6 t u y^{3}+\frac{6 u y^{3}}{t}+\frac{2 y^{3}}{t^{2}}+\frac{6 t y^{3}}{u}+\frac{6 y^{3}}{t u}+\frac{2 y^{3}}{u^{2}} \\
& +14 y^{3}-t^{3} y^{2}-u^{3} y^{2}-9 t u^{2} y^{2}-33 t y^{2}-9 t^{2} u y^{2}-33 u y^{2}-\frac{9 u^{2} y^{2}}{t}-\frac{33 y^{2}}{t}-\frac{9 u y^{2}}{t^{2}}-\frac{y^{2}}{t^{3}}-\frac{9 t^{2} y^{2}}{u} \\
& -\frac{33 y^{2}}{u}-\frac{9 y^{2}}{t^{2} u}-\frac{9 t y^{2}}{u^{2}}-\frac{9 y^{2}}{t u^{2}}-\frac{y^{2}}{u^{3}}+4 t u^{3} y+\frac{4 u^{3} y}{t}+28 t^{2} y+10 t^{2} u^{2} y+\frac{10 u^{2} y}{t^{2}}+28 u^{2} y+4 t^{3} u y \\
& +52 t u y+\frac{52 u y}{t}+\frac{4 u y}{t^{3}}+\frac{28 y}{t^{2}}+\frac{4 t^{3} y}{u}+\frac{52 t y}{u}+\frac{52 y}{t u}+\frac{4 y}{t^{3} u}+\frac{10 t^{2} y}{u^{2}}+\frac{28 y}{u^{2}}+\frac{10 y}{t^{2} u^{2}}+\frac{4 t y}{u^{3}}+\frac{4 y}{t u^{3}} \\
& +90 y-8 t^{3}-3 t^{2} u^{3}-8 u^{3}-3 t^{3} u^{2}-29 t u^{2}-86 t-29 t^{2} u-86 u-\frac{29 u^{2}}{t}-\frac{86}{t}-\frac{3 u^{3}}{t^{2}}-\frac{29 u}{t^{2}} \\
& -\frac{3 u^{2}}{t^{3}}-\frac{8}{t^{3}}-\frac{29 t^{2}}{u}-\frac{86}{u}-\frac{29}{t^{2} u}-\frac{3 t^{3}}{u^{2}}-\frac{29 t}{u^{2}}-\frac{29}{t u^{2}}-\frac{3}{t^{3} u^{2}}-\frac{3 t^{2}}{u^{3}}-\frac{8}{u^{3}}-\frac{3}{t^{2} u^{3}}+\frac{4 u^{3}}{t y}+\frac{52 u}{t y} \\
& +\frac{10 u^{2}}{t^{2} y}+\frac{28}{t^{2} y}+\frac{4 u}{t^{3} y}+\frac{4 t^{3}}{u y}+\frac{52 t}{u y}+\frac{52}{t u y}+\frac{4}{t^{3} u y}+\frac{10 t^{2}}{u^{2} y}+\frac{28}{u^{2} y}+\frac{10}{t^{2} u^{2} y}+\frac{4 t}{u^{3} y}+\frac{4}{t u^{3} y}-\frac{t^{3}}{y^{2}}
\end{aligned}
$$




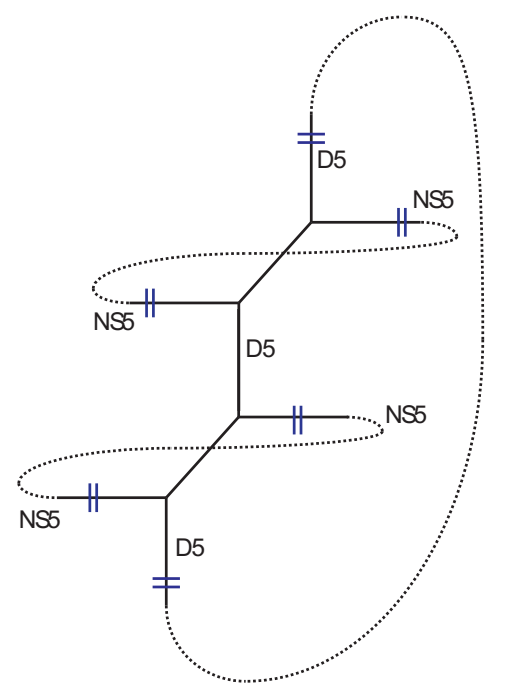

Figure 5. $(p, q)$ fivebranes web dual to rank 2 little string theory.

$$
\begin{aligned}
& -\frac{u^{3}}{y^{2}}-\frac{9 t u^{2}}{y^{2}}-\frac{33 t}{y^{2}}-\frac{9 t^{2} u}{y^{2}}-\frac{33 u}{y^{2}}-\frac{9 u^{2}}{t y^{2}}-\frac{33}{t y^{2}}-\frac{9 u}{t^{2} y^{2}}-\frac{1}{t^{3} y^{2}}-\frac{9 t^{2}}{u y^{2}}-\frac{33}{u y^{2}}-\frac{9}{t^{2} u y^{2}}-\frac{9 t}{u^{2} y^{2}} \\
& -\frac{9}{t u^{2} y^{2}}-\frac{1}{u^{3} y^{2}}+\frac{2 t^{2}}{y^{3}}+\frac{2 u^{2}}{y^{3}}+\frac{6 t u}{y^{3}}+\frac{14}{y^{3}}+\frac{6 u}{t y^{3}}+\frac{2}{t^{2} y^{3}}+\frac{6 t}{u y^{3}}+\frac{6}{t u y^{3}}+\frac{2}{u^{2} y^{3}}-\frac{t}{y^{4}}-\frac{u}{y^{4}}-\frac{1}{t y^{4}} \\
& -\frac{1}{u y^{4}}+\frac{4 t u^{3}}{y}+\frac{28 t^{2}}{y}+\frac{10 t^{2} u^{2}}{y}+\frac{28 u^{2}}{y}+\frac{4 t^{3} u}{y}+\frac{52 t u}{y}+\frac{90}{y}
\end{aligned}
$$

and further up to $F_{444}(t, u, y)$.

Let us define the following variables,

$$
\hat{w}=w y^{-1}, \quad \hat{v}_{1}=v_{1} y^{-1}, \hat{v}_{2}=v_{2} y^{-1}
$$

We can check $\hat{w} \leftrightarrow y$ exchange symmetry of the index. This is an analog of the triality exchanging $(\hat{q}, \hat{w}, y)$ at $N=1$. It can be understood as a geometric duality of the dual $(p, q)$-fivebrane web diagram figure 5 of the rank 2 little string theory. Namely, let us define the index

$$
\tilde{Z}=P E\left[2 I_{\text {com }} y\right] \hat{Z}_{\text {IIA }} .
$$

We find that $\tilde{Z}$ is invariant under the $\hat{w} \leftrightarrow y$ exchange, to some high orders in fugacities.

\subsection{Three NS5-branes}

The index of U(3) IIB little string theory is given by

$$
Z_{\mathrm{IIB}}\left(\alpha_{i}, \epsilon_{ \pm}, m ; q, w\right)=Z_{\text {pert }}^{\mathrm{IIB}}\left(\alpha_{i}, \epsilon_{ \pm}, m ; q\right) Z_{\text {inst }}^{\mathrm{IIB}}\left(\alpha_{i}, \epsilon_{ \pm}, m ; q, w\right)
$$

with

$$
\begin{aligned}
Z_{\mathrm{pert}}^{\mathrm{IIB}}= & P E\left[I_{+}\left(v_{1}+v_{2}+v_{1} v_{2}\right)+3 I_{+} \frac{q}{1-q}\right] \\
& \times P E\left[I_{+}\left(v_{1}+v_{1}^{-1}+v_{2}+v_{2}^{-1}+v_{1} v_{2}+v_{1}^{-1} v_{2}^{-1}\right) \frac{q}{1-q}\right]
\end{aligned}
$$




$$
=P E\left[I_{+} \frac{v_{1}+v_{2}+v_{3}+v_{1} v_{2}+v_{1} v_{2}+v_{2} v_{3}+3 v_{1} v_{2} v_{3}}{1-v_{1} v_{2} v_{3}}\right] .
$$

where $v_{1}=e^{2 \pi i \alpha_{12}}, v_{2}=e^{2 \pi i \alpha_{23}}$, and $v_{3}=q v_{1}^{-1} v_{2}^{-1} \cdot Z_{k}$ 's appearing in $Z_{\text {inst }}^{\text {IIB }}$ are obtained from eq. (2.7).

The index of the rank 3 IIA little string theory is given by

$$
\begin{aligned}
Z_{\mathrm{IIA}} & =Z_{\mathrm{extra}}(q)^{-1} Z_{\mathrm{mom}}^{\mathrm{IIA}}\left(\epsilon_{ \pm}, m ; w\right) \hat{Z}_{\mathrm{string}}^{\mathrm{IIA}}\left(\alpha_{i}, \epsilon_{ \pm}, m ; w, q\right) \\
& =Z_{\text {extra }}(q)^{-1} Z_{\mathrm{mom}}^{\mathrm{IIA}}\left(\epsilon_{ \pm}, m ; w\right) \sum_{n_{i}=0}^{\infty} v_{1}^{n_{1}} v_{2}^{n_{2}} v_{3}^{n_{3}} Z_{\text {string }}^{\left(n_{1}, n_{2}, n_{3}\right)}\left(\epsilon_{ \pm}, m ; w\right)
\end{aligned}
$$

$Z_{\text {extra }}(q)$ is given by

$$
Z_{\text {extra }}=P E\left[\frac{q}{1-q}\right]=P E\left[\frac{v_{1} v_{2} v_{3}}{1-v_{1} v_{2} v_{3}}\right]
$$

$Z_{\mathrm{mom}}^{\mathrm{IIA}}\left(\epsilon_{ \pm}, m ; w\right)$ is given by

$$
Z_{\mathrm{mom}}^{\mathrm{IIA}}\left(\epsilon_{ \pm}, m ; w\right)=P E\left[3 I_{-}\left(\epsilon_{ \pm}, m\right) \frac{w}{1-w}\right] .
$$

Note that $Z_{\text {string }}^{\left(n_{1}, n_{2}, n_{3}\right)}$ is invariant under the permutation of $n_{1}, n_{2}$ and $n_{3}$, from the symmetry of the quiver. The elliptic genera of the IIA fractional little strings, $Z_{\text {string }}^{\left(n_{1}, n_{2}, n_{3}\right)}$, are obtained from eq. (3.15). For example,

$$
\begin{aligned}
& Z_{\text {string }}^{(1,0,0)}\left(\epsilon_{ \pm}, m ; w\right)=\frac{\theta_{1}\left(w, m \pm \epsilon_{+}\right)}{\theta_{1}\left(w, \epsilon_{1}\right) \theta_{1}\left(w, \epsilon_{2}\right)}, Z_{\text {string }}^{(1,1,0)}\left(\epsilon_{ \pm}, m ; w\right)=\frac{\theta_{1}\left(w ; m \pm \epsilon_{-}\right) \theta_{1}\left(w ; m \pm \epsilon_{+}\right)}{\theta_{1}\left(w ; \epsilon_{1}\right)^{2} \theta_{1}\left(w ; \epsilon_{2}\right)^{2}} \\
& Z_{\text {string }}^{(2,0,0)}\left(\epsilon_{ \pm}, m ; w\right)=Z_{\text {string }}^{(1,0,0)} \cdot\left(\frac{\theta_{1}\left(w ; \epsilon_{+}+\epsilon_{1} \pm m\right)}{\theta_{1}\left(w ; 2 \epsilon_{1}\right) \theta_{1}\left(w ; \epsilon_{1}-\epsilon_{2}\right)}-\left(\epsilon_{1} \leftrightarrow \epsilon_{2}\right)\right) \\
& Z_{\text {string }}^{(1,1,1)}\left(\epsilon_{ \pm}, m ; w\right)=\frac{\theta_{1}\left(w, m \pm \epsilon_{-}\right)^{3}}{\theta_{1}\left(w, \epsilon_{1}\right)^{3} \theta_{1}\left(w, \epsilon_{2}\right)^{3}}
\end{aligned}
$$

We write the IIB/IIA indices as

$$
\begin{aligned}
& Z_{\mathrm{IIB}}\left(\alpha_{i}, \epsilon_{ \pm}, m ; w, v_{i}\right)=P E\left[I_{\mathrm{com}}(t, u) \sum_{i, j, k, l=0}^{\infty} F_{i j k l}^{\mathrm{IIB}}(t, u, y) w^{i} v_{1}^{j} v_{2}^{k} v_{3}^{l}\right], \\
& Z_{\mathrm{IIA}}\left(\alpha_{i}, \epsilon_{ \pm}, m ; w, v_{i}\right)=P E\left[I_{\mathrm{com}}(t, u) \sum_{i, j, k, l=0}^{\infty} F_{i j k l}^{\mathrm{IIA}}(t, u, y) w^{i} v_{1}^{j} v_{2}^{k} v_{3}^{l}\right],
\end{aligned}
$$

where $I_{\text {com }}$ is given by eq. (4.20). The coefficients $F_{i j k l}(t, u, y)$ are polynomials of $t, u$, and $y$, satisfying $F_{i j k l}=F_{i(j k l)}$.

T-duality implies that $F_{i j k l}^{\mathrm{IIA}}=F_{i j k l}^{\mathrm{IIB}} \equiv F_{i j k l}$. We checked this for higher orders of the fugacity variables. $F_{i j k l}$ are given by

$$
F_{0100}=-t-\frac{1}{t}+y+\frac{1}{y}, \quad F_{0200}=0, \quad F_{0110}=-t-\frac{1}{t}+y+\frac{1}{y}, \quad F_{0210}=0
$$


$F_{0111}=-3 t-\frac{3}{t}+3 y+\frac{3}{y}, \quad F_{0220}=0, \quad F_{0211}=-t-\frac{1}{t}+y+\frac{1}{y}$

$F_{0221}=-t-\frac{1}{t}+y+\frac{1}{y}, \quad F_{0222}=-3 t-\frac{3}{t}+3 y+\frac{3}{y}, \quad F_{1000}=-3 u-\frac{3}{u}+3 y+\frac{3}{y}$

$F_{1100}=-t^{2} u-\frac{t^{2}}{u}-\frac{u}{t^{2}}-\frac{1}{t^{2} u}+t^{2} y+\frac{t^{2}}{y}+\frac{y}{t^{2}}+\frac{1}{t^{2} y}+t u y+\frac{t y}{u}+\frac{t u}{y}+\frac{t}{u y}+\frac{y}{t u}+\frac{u}{t y}+\frac{1}{t u y}$

$+\frac{u y}{t}-t y^{2}-\frac{t}{y^{2}}-\frac{1}{t y^{2}}-\frac{y^{2}}{t}-2 t-\frac{2}{t}-2 u-\frac{2}{u}+2 y+\frac{2}{y}$

$F_{1200}=-t^{4} u-\frac{t^{4}}{u}-\frac{u}{t^{4}}-\frac{1}{t^{4} u}+t^{4} y+\frac{t^{4}}{y}+\frac{y}{t^{4}}+\frac{1}{t^{4} y}+t^{3} u y+\frac{t^{3} y}{u}+\frac{t^{3} u}{y}+\frac{t^{3}}{u y}+\frac{u y}{t^{3}}+\frac{y}{t^{3} u}$

$+\frac{u}{t^{3} y}+\frac{1}{t^{3} u y}-t^{3} y^{2}-\frac{t^{3}}{y^{2}}-\frac{y^{2}}{t^{3}}-\frac{1}{t^{3} y^{2}}-2 t^{3}-\frac{2}{t^{3}}-2 t^{2} u-\frac{2 t^{2}}{u}-\frac{2 u}{t^{2}}-\frac{2}{t^{2} u}+2 t^{2} y$

$+\frac{2 t^{2}}{y}+\frac{2 y}{t^{2}}+\frac{2}{t^{2} y}+t u y+\frac{t y}{u}+\frac{t u}{y}+\frac{t}{u y}+\frac{y}{t u}+\frac{u}{t y}+\frac{1}{t u y}+\frac{u y}{t}-t y^{2}-\frac{t}{y^{2}}-\frac{1}{t y^{2}}$

$-\frac{y^{2}}{t}-2 t-\frac{2}{t}-2 u-\frac{2}{u}+2 y+\frac{2}{y}$

$F_{1110}=-2 t^{2} u-\frac{2 u}{t^{2}}-\frac{2 t^{2}}{u}-\frac{2}{t^{2} u}+2 t^{2} y+\frac{2 y}{t^{2}}+\frac{2}{t^{2} y}+\frac{2 t^{2}}{y}+3 t u y+\frac{3 u y}{t}+\frac{3 t y}{u}+\frac{3 y}{t u}+\frac{3 u}{t y}$

$+\frac{3 t}{u y}+\frac{3}{t u y}+\frac{3 t u}{y}-3 t y^{2}-\frac{3 y^{2}}{t}-\frac{3 t}{y^{2}}-\frac{3}{t y^{2}}-6 t-\frac{6}{t}-u y^{2}-\frac{y^{2}}{u}-\frac{u}{y^{2}}-\frac{1}{u y^{2}}-6 u$

$-\frac{6}{u}+y^{3}+\frac{1}{y^{3}}+7 y+\frac{7}{y}$

$F_{1210}=-t^{4} u-\frac{t^{4}}{u}-\frac{u}{t^{4}}-\frac{1}{t^{4} u}+t^{4} y+\frac{t^{4}}{y}+\frac{y}{t^{4}}+\frac{1}{t^{4} y}+2 t^{3} u y+\frac{2 t^{3} y}{u}+\frac{2 t^{3} u}{y}+\frac{2 t^{3}}{u y}+\frac{2 u y}{t^{3}}+\frac{2 y}{t^{3} u}$

$+\frac{2 u}{t^{3} y}+\frac{2}{t^{3} u y}-2 t^{3} y^{2}-\frac{2 t^{3}}{y^{2}}-\frac{2 y^{2}}{t^{3}}-\frac{2}{t^{3} y^{2}}-4 t^{3}-\frac{4}{t^{3}}-t^{2} u y^{2}-\frac{t^{2} y^{2}}{u}-\frac{t^{2} u}{y^{2}}-\frac{t^{2}}{u y^{2}}-\frac{u y^{2}}{t^{2}}$

$-\frac{y^{2}}{t^{2} u}-\frac{u}{t^{2} y^{2}}-\frac{1}{t^{2} u y^{2}}-5 t^{2} u-\frac{5 t^{2}}{u}-\frac{5 u}{t^{2}}-\frac{5}{t^{2} u}+t^{2} y^{3}+\frac{t^{2}}{y^{3}}+\frac{y^{3}}{t^{2}}+\frac{1}{t^{2} y^{3}}+6 t^{2} y+\frac{6 t^{2}}{y}$

$+\frac{6 y}{t^{2}}+\frac{6}{t^{2} y}+4 t u y+\frac{4 t y}{u}+\frac{4 t u}{y}+\frac{4 t}{u y}+\frac{4 y}{t u}+\frac{4 u}{t y}+\frac{4}{t u y}+\frac{4 u y}{t}-4 t y^{2}-\frac{4 t}{y^{2}}-\frac{4}{t y^{2}}-\frac{4 y^{2}}{t}$

$-8 t-\frac{8}{t}-u y^{2}-\frac{y^{2}}{u}-\frac{u}{y^{2}}-\frac{1}{u y^{2}}-6 u-\frac{6}{u}+y^{3}+\frac{1}{y^{3}}+7 y+\frac{7}{y}$

$F_{1111}=-6 t^{2} u-\frac{6 u}{t^{2}}-\frac{6 t^{2}}{u}-\frac{6}{t^{2} u}+6 t^{2} y+\frac{6 y}{t^{2}}+\frac{6}{t^{2} y}+\frac{6 t^{2}}{y}-3 t u^{2}-\frac{3 u^{2}}{t}-\frac{3 t}{u^{2}}-\frac{3}{t u^{2}}+15 t u y$

$+\frac{15 u y}{t}+\frac{15 t y}{u}+\frac{15 y}{t u}+\frac{15 u}{t y}+\frac{15 t}{u y}+\frac{15}{t u y}+\frac{15 t u}{y}-12 t y^{2}-\frac{12 y^{2}}{t}-\frac{12 t}{y^{2}}-\frac{12}{t y^{2}}-30 t$

$-\frac{30}{t}+3 u^{2} y+\frac{3 y}{u^{2}}+\frac{3}{u^{2} y}+\frac{3 u^{2}}{y}-9 u y^{2}-\frac{9 y^{2}}{u}-\frac{9 u}{y^{2}}-\frac{9}{u y^{2}}-30 u-\frac{30}{u}+6 y^{3}+\frac{6}{y^{3}}$

$+36 y+\frac{36}{y}$

$F_{1220}=-2 t^{4} u-\frac{2 t^{4}}{u}-\frac{2 u}{t^{4}}-\frac{2}{t^{4} u}+2 t^{4} y+\frac{2 t^{4}}{y}+\frac{2 y}{t^{4}}+\frac{2}{t^{4} y}+3 t^{3} u y+\frac{3 t^{3} y}{u}+\frac{3 t^{3} u}{y}+\frac{3 t^{3}}{u y}+\frac{3 u y}{t^{3}}$

$+\frac{3 y}{t^{3} u}+\frac{3 u}{t^{3} y}+\frac{3}{t^{3} u y}-3 t^{3} y^{2}-\frac{3 t^{3}}{y^{2}}-\frac{3 y^{2}}{t^{3}}-\frac{3}{t^{3} y^{2}}-6 t^{3}-\frac{6}{t^{3}}-t^{2} u y^{2}-\frac{t^{2} y^{2}}{u}-\frac{t^{2} u}{y^{2}}-\frac{t^{2}}{u y^{2}}$

$-\frac{u y^{2}}{t^{2}}-\frac{y^{2}}{t^{2} u}-\frac{u}{t^{2} y^{2}}-\frac{1}{t^{2} u y^{2}}-8 t^{2} u-\frac{8 t^{2}}{u}-\frac{8 u}{t^{2}}-\frac{8}{t^{2} u}+t^{2} y^{3}+\frac{t^{2}}{y^{3}}+\frac{y^{3}}{t^{2}}+\frac{1}{t^{2} y^{3}}+9 t^{2} y$

$+\frac{9 t^{2}}{y}+\frac{9 y}{t^{2}}+\frac{9}{t^{2} y}+7 t u y+\frac{7 t y}{u}+\frac{7 t u}{y}+\frac{7 t}{u y}+\frac{7 y}{t u}+\frac{7 u}{t y}+\frac{7}{t u y}+\frac{7 u y}{t}-7 t y^{2}-\frac{7 t}{y^{2}}-\frac{7}{t y^{2}}$

$-\frac{7 y^{2}}{t}-14 t-\frac{14}{t}-2 u y^{2}-\frac{2 y^{2}}{u}-\frac{2 u}{y^{2}}-\frac{2}{u y^{2}}-12 u-\frac{12}{u}+2 y^{3}+\frac{2}{y^{3}}+14 y+\frac{14}{y}$ 


$$
\begin{aligned}
& F_{1211}=-2 u t^{4}+2 y t^{4}-\frac{2 t^{4}}{u}+\frac{2 t^{4}}{y}-u^{2} t^{3}-5 y^{2} t^{3}+6 u y t^{3}+\frac{6 y t^{3}}{u}-\frac{t^{3}}{u^{2}}+\frac{6 u t^{3}}{y}+\frac{6 t^{3}}{u y}-\frac{5 t^{3}}{y^{2}} \\
& -12 t^{3}+4 y^{3} t^{2}-6 u y^{2} t^{2}-22 u t^{2}+2 u^{2} y t^{2}+\frac{2 y t^{2}}{u^{2}}+26 y t^{2}-\frac{6 y^{2} t^{2}}{u}-\frac{22 t^{2}}{u}+\frac{2 u^{2} t^{2}}{y}+\frac{26 t^{2}}{y} \\
& +\frac{2 t^{2}}{u^{2} y}-\frac{6 u t^{2}}{y^{2}}-\frac{6 t^{2}}{u y^{2}}+\frac{4 t^{2}}{y^{3}}-y^{4} t+2 u y^{3} t+\frac{2 y^{3} t}{u}-5 u^{2} t-u^{2} y^{2} t-24 y^{2} t+27 u y t+\frac{27 y t}{u} \\
& -\frac{y^{2} t}{u^{2}}-\frac{5 t}{u^{2}}+\frac{27 u t}{y}+\frac{27 t}{u y}-\frac{u^{2} t}{y^{2}}-\frac{24 t}{y^{2}}-\frac{t}{u^{2} y^{2}}+\frac{2 u t}{y^{3}}+\frac{2 t}{u y^{3}}-\frac{t}{y^{4}}-52 t+\frac{4 y^{3}}{t^{2}}+\frac{2 y^{3}}{t u} \\
& +9 y^{3}-13 u y^{2}-42 u+\frac{2 u^{2} y}{t^{2}}+4 u^{2} y+\frac{6 u y}{t^{3}}+\frac{26 y}{t^{2}}+\frac{2 y}{t^{4}}+\frac{27 y}{t u}+\frac{6 y}{t^{3} u}+\frac{4 y}{u^{2}}+\frac{2 y}{t^{2} u^{2}}+51 y \\
& -\frac{6 u y^{2}}{t^{2}}-\frac{22 u}{t^{2}}-\frac{u^{2}}{t^{3}}-\frac{5 y^{2}}{t^{3}}-\frac{12}{t^{3}}-\frac{2 u}{t^{4}}-\frac{13 y^{2}}{u}-\frac{42}{u}-\frac{6 y^{2}}{t^{2} u}-\frac{22}{t^{2} u}-\frac{2}{t^{4} u}-\frac{y^{2}}{t u^{2}}-\frac{5}{t u^{2}} \\
& -\frac{1}{t^{3} u^{2}}+\frac{4 u^{2}}{y}+\frac{51}{y}+\frac{27 u}{t y}+\frac{2 u^{2}}{t^{2} y}+\frac{26}{t^{2} y}+\frac{6 u}{t^{3} y}+\frac{2}{t^{4} y}+\frac{27}{t u y}+\frac{6}{t^{3} u y}+\frac{4}{u^{2} y}+\frac{2}{t^{2} u^{2} y}-\frac{13 u}{y^{2}} \\
& -\frac{u^{2}}{t y^{2}}-\frac{24}{t y^{2}}-\frac{6 u}{t^{2} y^{2}}-\frac{5}{t^{3} y^{2}}-\frac{13}{u y^{2}}-\frac{6}{t^{2} u y^{2}}-\frac{1}{t u^{2} y^{2}}+\frac{9}{y^{3}}+\frac{2 u}{t y^{3}}+\frac{4}{t^{2} y^{3}}+\frac{2}{t u y^{3}}-\frac{1}{t y^{4}} \\
& -\frac{y^{4}}{t}+\frac{2 u y^{3}}{t}-\frac{5 u^{2}}{t}-\frac{u^{2} y^{2}}{t}-\frac{24 y^{2}}{t}+\frac{27 u y}{t}-\frac{52}{t} \\
& F_{2000}=-3 u-\frac{3}{u}+3 y+\frac{3}{y} \\
& F_{2100}=-t^{3} u^{2}-\frac{t^{3}}{u^{2}}-\frac{u^{2}}{t^{3}}-\frac{1}{t^{3} u^{2}}+t^{3} u y+\frac{t^{3} y}{u}+\frac{t^{3} u}{y}+\frac{t^{3}}{u y}+\frac{u y}{t^{3}}+\frac{y}{t^{3} u}+\frac{u}{t^{3} y}+\frac{1}{t^{3} u y}-2 t^{3} \\
& -\frac{2}{t^{3}}+t^{2} u^{2} y+\frac{t^{2} y}{u^{2}}+\frac{t^{2} u^{2}}{y}+\frac{t^{2}}{u^{2} y}+\frac{u^{2} y}{t^{2}}+\frac{y}{t^{2} u^{2}}+\frac{u^{2}}{t^{2} y}+\frac{1}{t^{2} u^{2} y}-t^{2} u y^{2}-\frac{t^{2} y^{2}}{u}-\frac{t^{2} u}{y^{2}} \\
& -\frac{t^{2}}{u y^{2}}-\frac{u y^{2}}{t^{2}}-\frac{y^{2}}{t^{2} u}-\frac{u}{t^{2} y^{2}}-\frac{1}{t^{2} u y^{2}}-3 t^{2} u-\frac{3 t^{2}}{u}-\frac{3 u}{t^{2}}-\frac{3}{t^{2} u}+3 t^{2} y+\frac{3 t^{2}}{y}+\frac{3 y}{t^{2}}+\frac{3}{t^{2} y} \\
& -2 t u^{2}-\frac{2 t}{u^{2}}-\frac{2}{t u^{2}}-\frac{2 u^{2}}{t}+4 t u y+\frac{4 t y}{u}+\frac{4 t u}{y}+\frac{4 t}{u y}+\frac{4 y}{t u}+\frac{4 u}{t y}+\frac{4}{t u y}+\frac{4 u y}{t}-2 t y^{2}-\frac{2 t}{y^{2}} \\
& -\frac{2}{t y^{2}}-\frac{2 y^{2}}{t}-8 t-\frac{8}{t}+u^{2} y+\frac{y}{u^{2}}+\frac{u^{2}}{y}+\frac{1}{u^{2} y}-2 u y^{2}-\frac{2 y^{2}}{u}-\frac{2 u}{y^{2}}-\frac{2}{u y^{2}}-6 u-\frac{6}{u} \\
& +y^{3}+\frac{1}{y^{3}}+7 y+\frac{7}{y}
\end{aligned}
$$

$$
\begin{aligned}
F_{2110}= & -t y^{4}-\frac{y^{4}}{t}+2 t^{2} y^{3}+3 t u y^{3}+\frac{3 u y^{3}}{t}+\frac{2 y^{3}}{t^{2}}+\frac{3 t y^{3}}{u}+\frac{3 y^{3}}{t u}+7 y^{3}-t^{3} y^{2}-2 t u^{2} y^{2}-17 t y^{2} \\
& -7 t^{2} u y^{2}-16 u y^{2}-\frac{2 u^{2} y^{2}}{t}-\frac{17 y^{2}}{t}-\frac{7 u y^{2}}{t^{2}}-\frac{y^{2}}{t^{3}}-\frac{7 t^{2} y^{2}}{u}-\frac{16 y^{2}}{u}-\frac{7 y^{2}}{t^{2} u}-\frac{2 t y^{2}}{u^{2}}-\frac{2 y^{2}}{t u^{2}} \\
& +19 t^{2} y+5 t^{2} u^{2} y+\frac{5 u^{2} y}{t^{2}}+9 u^{2} y+4 t^{3} u y+26 t u y+\frac{26 u y}{t}+\frac{4 u y}{t^{3}}+\frac{19 y}{t^{2}}+\frac{4 t^{3} y}{u}+\frac{26 t y}{u} \\
& +\frac{26 y}{t u}+\frac{4 y}{t^{3} u}+\frac{5 t^{2} y}{u^{2}}+\frac{9 y}{u^{2}}+\frac{5 y}{t^{2} u^{2}}+45 y-8 t^{3}-3 t^{3} u^{2}-12 t u^{2}-48 t-17 t^{2} u-38 u-\frac{12 u^{2}}{t} \\
& -\frac{48}{t}-\frac{17 u}{t^{2}}-\frac{3 u^{2}}{t^{3}}-\frac{8}{t^{3}}-\frac{17 t^{2}}{u}-\frac{38}{u}-\frac{17}{t^{2} u}-\frac{3 t^{3}}{u^{2}}-\frac{12 t}{u^{2}}-\frac{12}{t u^{2}}-\frac{3}{t^{3} u^{2}}+\frac{26 u}{t y}+\frac{5 u^{2}}{t^{2} y}+\frac{19}{t^{2} y} \\
& +\frac{4 u}{t^{3} y}+\frac{4 t^{3}}{u y}+\frac{26 t}{u y}+\frac{26}{t u y}+\frac{4}{t^{3} u y}+\frac{5 t^{2}}{u^{2} y}+\frac{9}{u^{2} y}+\frac{5}{t^{2} u^{2} y}-\frac{t^{3}}{y^{2}}-\frac{2 t u^{2}}{y^{2}}-\frac{17 t}{y^{2}}-\frac{7 t^{2} u}{y^{2}}-\frac{16 u}{y^{2}} \\
& -\frac{2 u^{2}}{t y^{2}}-\frac{17}{t y^{2}}-\frac{7 u}{t^{2} y^{2}}-\frac{1}{t^{3} y^{2}}-\frac{7 t^{2}}{u y^{2}}-\frac{16}{u y^{2}}-\frac{7}{t^{2} u y^{2}}-\frac{2 t}{u^{2} y^{2}}-\frac{2}{t u^{2} y^{2}}+\frac{2 t^{2}}{y^{3}}+\frac{3 t u}{y^{3}}+\frac{7}{y^{3}}+\frac{3 u}{t y^{3}} \\
& +\frac{2}{t^{2} y^{3}}+\frac{3 t}{u y^{3}}+\frac{3}{t u y^{3}}-\frac{t}{y^{4}}-\frac{1}{t y^{4}}+\frac{19 t^{2}}{y}+\frac{5 t^{2} u^{2}}{y}+\frac{9 u^{2}}{y}+\frac{4 t^{3} u}{y}+\frac{26 t u}{y}+\frac{45}{y}
\end{aligned}
$$

and so on.

For the rank 3 indices, we can also check the duality of exchanging $\hat{w}=w y^{-1}$ and $y$. 


\section{$5 \operatorname{SL}(2, Z)$ transformations of the elliptic genus}

The index of the winding IIB little strings is given by

$$
Z_{\text {inst }}^{\mathrm{IIB}}\left(\alpha_{i}, \epsilon_{ \pm}, m ; q, w\right)=\sum_{k=1}^{\infty} w^{k} \sum_{Y: \sum_{i}\left|Y_{i}\right|=k} \prod_{i, j=1}^{N} \prod_{s \in Y_{i}} \frac{\theta_{1}\left(q ; E_{i j}+m-\epsilon_{-}\right) \theta_{1}\left(q ; E_{i j}-m-\epsilon_{-}\right)}{\theta_{1}\left(q ; E_{i j}-\epsilon_{1}\right) \theta_{1}\left(q ; E_{i j}+\epsilon_{2}\right)}
$$

where

$$
E_{i j}=\alpha_{i}-\alpha_{j}-\epsilon_{1} h_{i}(s)+\epsilon_{2} v_{j}(s) .
$$

$q$ and $w$ are given by

$$
q=e^{2 \pi i \tau}, w=e^{2 \pi i \rho},
$$

where $\tau=i \frac{R_{\beta}}{R_{\mathrm{IIB}}}$ is the complex structure on the torus, $\alpha^{\prime} \rho=i R_{\beta} R_{\mathrm{IIB}}$ is the Kähler parameter of it, and $R_{\beta}$ is the radius of the temporal circle.

The modular transformation of the Jacobi's theta function is given by

$$
\theta_{1}\left(-\frac{1}{\tau} ; \frac{z}{\tau}\right)=-i\left(-i \tau^{\frac{1}{2}}\right) \exp \left(\frac{i \pi z^{2}}{\tau}\right) \theta_{1}(\tau ; z)
$$

Using this property, the S-duality transformation of $Z_{\text {inst }}^{\mathrm{IIB}}$ in $\tau$ is given by

$$
\sum_{k=1}^{\infty} w^{k} \exp \left(-2 \pi i \frac{m^{2}-\epsilon_{+}^{2}}{\tau} k N\right) \sum_{Y: \sum_{i}\left|Y_{i}\right|=k} \prod_{i, j=1}^{N} \prod_{s \in Y_{i}} \frac{\theta_{1}\left(-\frac{1}{\tau} ; \frac{E_{i j}+m-\epsilon_{-}}{\tau}\right) \theta_{1}\left(-\frac{1}{\tau} ; \frac{E_{i j}-m-\epsilon_{-}}{\tau}\right)}{\theta_{1}\left(-\frac{1}{\tau} ; \frac{E_{i j}-\epsilon_{1}}{\tau}\right) \theta_{1}\left(-\frac{1}{\tau} ; \frac{E_{i j}+\epsilon_{2}}{\tau}\right)} .
$$

Transforming the fugacity variable for the winding number, $w$ by

$$
w \rightarrow \tilde{w}=e^{-2 \pi i \frac{m^{2}-\epsilon_{+}^{2}}{\tau} N} w,
$$

$Z_{\text {inst }}^{\mathrm{IIB}}$ is invariant under the following transformation,

$$
q=e^{2 \pi i \tau} \rightarrow \tilde{q}=e^{-\frac{2 \pi i}{\tau}}, w \rightarrow \tilde{w}=e^{-2 \pi i \frac{m^{2}-\epsilon_{+}^{2}}{\tau} N} w .
$$

The elliptic genus of the IIA strings is given by

$$
\begin{aligned}
\hat{Z}_{\text {string }}^{\mathrm{IIA}}\left(\alpha_{i}, \epsilon_{ \pm}, m ; q^{\prime}, w^{\prime}\right) & =\sum_{n_{i}=0}^{\infty} e^{2 \pi i \sum_{i=1}^{N} n_{i} \alpha_{i, i+1}} Z_{\text {string }}^{\left(n_{1}, \ldots, n_{N}\right)}\left(\epsilon_{ \pm}, m ; q^{\prime}\right) \\
& =\sum_{n_{i}=0}^{\infty}\left(v_{1}\right)^{n_{1}} \cdots\left(v_{N}\right)^{n_{N}} Z_{\text {string }}^{\left(n_{1}, \ldots, n_{N}\right)}\left(\epsilon_{ \pm}, m ; q^{\prime}\right) \\
& =\sum_{n_{i}=0}^{\infty}\left(v_{1}\right)^{n_{1}-n_{N}} \cdots\left(v_{N-1}\right)^{n_{N-1}-n_{N}}\left(w^{\prime}\right)^{n_{N}} Z_{\text {string }}^{\left(n_{1}, \ldots, n_{N}\right)}\left(\epsilon_{ \pm}, m ; q^{\prime}\right)
\end{aligned}
$$

where $v_{i}=e^{2 \pi i\left(\alpha_{i, i+1}\right)}$ and $e^{-2 \pi i\left(\alpha_{N+1}\right)}=e^{-2 \pi i \alpha_{1}} w^{\prime} . Z_{\text {string }}^{\left(n_{1}, \ldots, n_{N}\right)}$ is given by

$$
Z_{\text {string }}^{\left(n_{1}, \ldots, n_{N}\right)}\left(\epsilon_{ \pm}, m ; q^{\prime}\right)=\sum_{\left\{Y_{1}, \cdots, Y_{N}\right\} ;\left|Y_{i}\right|=n_{i}} \prod_{i=1}^{N} \prod_{(a, b) \in Y_{i}} \frac{\theta_{1}\left(q^{\prime} ; E_{i, i+1}^{(a, b)}-m+\epsilon_{-}\right) \theta_{1}\left(q^{\prime} ; E_{i, i-1}^{(a, b)}+m+\epsilon_{-}\right)}{\theta_{1}\left(q^{\prime} ; E_{i, i}^{(a, b)}+\epsilon_{1}\right) \theta_{1}\left(q^{\prime} ; E_{i, i}^{(a, b)}-\epsilon_{2}\right)},
$$


where

$$
E_{i j}^{(a, b)}=\left(Y_{i, a}-b\right) \epsilon_{1}-\left(Y_{j, b}^{T}-a\right) \epsilon_{2} .
$$

Upon T-duality transformation, the complex structure and the Kähler parameter are exchanged. The modular transformation of $Z_{\text {string }}^{\left(n_{1}, \ldots, n_{N}\right)}$ in $\rho \equiv \tau^{\prime}$ is given by

$$
\begin{aligned}
& Z_{\text {string }}^{\left(n_{1}, \cdots, n_{N}\right)}\left(\epsilon_{ \pm}, m ; \rho\right) \\
& \quad=\exp \left[-\frac{\pi i}{\rho}\left(\epsilon_{1} \epsilon_{2} \sum_{a=1}^{N}\left(n_{a}-n_{a+1}\right)^{2}+2\left(m^{2}-\epsilon_{+}^{2}\right) \sum_{a=1}^{N} n_{a}\right)\right] \cdot Z_{\text {string }}^{\left(n_{1}, \cdots, n_{N}\right)}\left(\frac{\epsilon_{ \pm}}{\rho}, \frac{m}{\rho} ;-\frac{1}{\rho}\right) .
\end{aligned}
$$

where $n_{N+1}=n_{1}$. Via T-duality relation, this would imply a definite S-duality transformation of $Z^{\mathrm{IIB}}$ in $\rho$, which would have been difficult to obtain directly without knowing the T-dual expression. Note that the above S-duality transformation becomes paraticularly simpler when $n_{1}=n_{2}=\cdots=n_{N}$ :

$$
Z_{\text {string }}^{(n, \cdots, n)}\left(\epsilon_{ \pm}, m ; \rho\right)=\exp \left[-\frac{2 \pi i}{\rho}\left(m^{2}-\epsilon_{+}^{2}\right) N n\right] \cdot Z_{\text {string }}^{\left(n_{1}, \cdots, n_{N}\right)}\left(\frac{\epsilon_{ \pm}}{\rho}, \frac{m}{\rho} ;-\frac{1}{\rho}\right) .
$$

The prefactor can be absorbed into a scaling of $w^{\prime}=q$ fugacity, conjugate to $n$. This expression might be useful to understand the DLCQ of type IIB little strings, in which $\mathrm{U}(n)^{N}$ gauge theory description was used [7].

\section{Concluding remarks}

In this paper, we explored the 2 dimensional $\mathcal{N}=(4,4)$ and $\mathcal{N}=(0,4)$ gauge theory descriptions of macroscopic IIA/IIB little strings. In particular, we proposed a new $(0,4)$ gauge theory which enables the computation of the IIA strings' elliptic genera. We used these elliptic genera to study the little string T-duality.

The elliptic genus is enjoying $\mathrm{SL}(2, \mathbb{Z}) \times \mathrm{SL}(2, \mathbb{Z})$ symmetry on the complex structure $\tau$ and Kahler parameter $\rho$ of the torus. Interesting extended dualities were studied in [25] for 6d maximal SYM theory compactified on $T^{2}$, from its Seiberg-Witten curve. It will be interesting to see whether a larger duality than what we explored here is realized in the elliptic genera.

It will also be interesting to see if one can study the T-duality of elliptic genera for the heterotic little string theories, living on the heterotic 5-branes in the $\mathrm{SO}(32)$ and $E_{8} \times E_{8}$ theories. Just like our IIA strings are closely related to the 'M-strings' of $6 \mathrm{~d}(2,0) \mathrm{CFT}$, the $E_{8} \times E_{8}$ little strings would be closely related to the so-called E-strings of the $6 \mathrm{~d}(1,0)$ CFT, with $E_{8}$ global symmetry [30,31]. The E-string elliptic genera have been recently studied in [32], from $2 \mathrm{~d}(0,4)$ gauge theories.

Finally, the self-dual string elliptic genera in 6d SCFTs turn out to be related to other interesting observables, such as the superconformal indices [33-38]. It will be interesting to see if the elliptic genera for little strings also find similar interesting applications. 


\section{Acknowledgments}

This work is supported in part by the National Research Foundation of Korea (NRF) Grants No. 2012R1A1A2042474 (JK,SK), 2012R1A2A2A02046739 (SK), 2006-0093850 (KL), 2009-0084601 (KL), NRF-2015R1A2A2A01003124 (SK).

\section{A $\mathcal{N}=(4,4)$ gauge theory of IIB strings}

The Lagrangian of the $2 \mathrm{~d} N=(4,4)$ gauge theory for IIB strings is given by

$$
\mathcal{L}=\mathcal{L}_{1}+\mathcal{L}_{2}
$$

$\mathcal{L}_{1}$ is given by

$$
\begin{aligned}
\mathcal{L}_{1}= & \frac{1}{g_{Q M}^{2}} \operatorname{Tr}\left[-\frac{1}{4}\left(F_{\mu \nu}\right)^{2}-\frac{1}{2}\left(D_{\mu} \varphi_{a A}\right)\left(D^{\mu} \varphi^{A a}\right)-\frac{1}{2}\left(D_{\mu} a_{\alpha \dot{\beta}}\right)\left(D^{\mu} a^{\dot{\beta} \alpha}\right)+\frac{1}{2}\left[a_{\alpha \dot{\beta}}, \varphi_{a A}\right]^{2}\right. \\
& +\frac{i}{2}\left(\bar{\lambda}_{a}^{\dot{\alpha}}\right)^{\dagger}\left(D_{t}+D_{s}\right) \bar{\lambda}_{a}^{\dot{\alpha}}+\frac{i}{2}\left(\bar{\lambda}^{A \dot{\alpha}}\right)^{\dagger}\left(D_{t}-D_{s}\right) \bar{\lambda}^{A \dot{\alpha}}+\frac{i}{2}\left(\lambda_{\alpha}^{A}\right)^{\dagger}\left(D_{t}+D_{s}\right) \lambda_{\alpha}^{A}+\frac{i}{2}\left(\lambda_{a \alpha}\right)^{\dagger}\left(D_{t}-D_{s}\right) \lambda_{a \alpha} \\
& +\frac{1}{2} D^{I} D^{I}-D^{I}\left(\bar{q}^{\dot{\alpha}} q_{\dot{\beta}}\left(\tau^{I}\right)^{\dot{\alpha}}{ }_{\dot{\beta}}+\frac{1}{2}\left(\tau^{I}\right)^{\dot{\alpha}} \dot{\dot{\beta}}\left[a^{\dot{\beta} \alpha}, a_{\alpha \dot{\alpha}}\right]-\zeta^{I}\right)+\frac{1}{2} D^{I^{\prime}} D^{I^{\prime}}-D^{I^{\prime}}\left(\frac{1}{2}\left(\tau^{I^{\prime}}\right)^{A}{ }_{B}\left[\varphi^{B a}, \varphi_{a A}\right]\right) \\
& -\frac{i}{\sqrt{2}}\left(\lambda_{a \alpha}\right)^{\dagger}\left[a_{\alpha \dot{\beta}}, \bar{\lambda}_{a}^{\dot{\beta}}\right]-\frac{i}{\sqrt{2}}\left(\lambda_{\alpha}^{A}\right)^{\dagger}\left[a_{\alpha \dot{\beta}}, \bar{\lambda}^{A \dot{\beta}}\right]+\frac{i}{\sqrt{2}}\left(\bar{\lambda}^{A \dot{\alpha}}\right)^{\dagger}\left[a^{\dot{\alpha} \beta}, \lambda_{\beta}^{A}\right]+\frac{i}{\sqrt{2}}\left(\bar{\lambda}_{a}^{\dot{\alpha}}\right)^{\dagger}\left[a^{\dot{\alpha} \beta}, \lambda_{a \beta}\right] \\
& \left.+\frac{i}{\sqrt{2}}\left(\bar{\lambda}_{a}^{\dot{\alpha}}\right)^{\dagger}\left[\varphi_{a A}, \bar{\lambda}^{A \dot{\alpha}}\right]+\frac{i}{\sqrt{2}}\left(\bar{\lambda}^{A \dot{\alpha}}\right)^{\dagger}\left[\varphi^{A a}, \bar{\lambda}_{a}^{\dot{\alpha}}\right]-\frac{i}{\sqrt{2}}\left(\lambda_{a \alpha}\right)^{\dagger}\left[\varphi_{a A}, \lambda_{\alpha}^{A}\right]-\frac{i}{\sqrt{2}}\left(\lambda_{\alpha}^{A}\right)^{\dagger}\left[\varphi^{A a}, \lambda_{a \alpha}\right]\right] .
\end{aligned}
$$

$\mathcal{L}_{2}$ is given by,

$$
\begin{aligned}
\mathcal{L}_{2}= & \operatorname{Tr}\left[-D_{\mu} \bar{q}^{\dot{\alpha}} D_{\mu} q_{\dot{\alpha}}-\varphi_{a A} \bar{q}^{\dot{\alpha}} q_{\dot{\alpha}} \varphi^{A a}+i\left(\psi_{a}\right)^{\dagger}\left(D_{t}-D_{s}\right) \psi_{a}+i\left(\psi^{A}\right)^{\dagger}\left(D_{t}+D_{s}\right) \psi^{A}\right. \\
& +\sqrt{2}\left(\psi_{a}\right)^{\dagger}\left(\psi^{A} \varphi_{a A}\right)+\sqrt{2}\left(\psi^{A}\right)^{\dagger}\left(\psi_{a} \varphi^{A a}\right)+i \sqrt{2}\left(\lambda_{a}^{\dot{\alpha}}\right)^{\dagger} \bar{q}^{\dot{\alpha}} \psi_{a}+i \sqrt{2}\left(\bar{\lambda}^{A \dot{\alpha}}\right)^{\dagger} \bar{q}^{\dot{\alpha}} \psi^{A} \\
& \left.\left.-i \sqrt{2}\left(\psi_{a}\right)^{\dagger} q_{\dot{\alpha}} \bar{\lambda}_{a}^{\dot{\alpha}}-i \sqrt{2}\left(\psi^{A}\right)^{\dagger} q_{\dot{\alpha}} \bar{\lambda}^{A \dot{\alpha}}\right)\right] .
\end{aligned}
$$

In the Higgs branch, the theory describes IIB strings bound to the NS5-branes, whose target space is the $k$ instanton moduli space.

The reality condition of the scalar fields is given by

$$
\begin{array}{rlrl}
a_{\alpha \dot{\alpha}} & =\frac{1}{\sqrt{2}}\left(\sigma^{m}\right)_{\alpha \dot{\alpha}} a_{m}, & a^{\dot{\alpha} \alpha}=\frac{1}{\sqrt{2}}\left(\bar{\sigma}^{m}\right)^{\dot{\alpha} \alpha} a_{m}, & a^{\dot{\alpha} \alpha}=\epsilon^{\alpha \beta} \epsilon^{\dot{\alpha} \dot{\beta}} a_{\beta \dot{\beta}}=\left(a_{\alpha \dot{\alpha}}\right)^{\dagger}, \\
\varphi_{a A}=\frac{1}{\sqrt{2}}\left(\sigma^{I}\right)_{a A} \varphi_{I}, & \varphi^{A a}=\frac{1}{\sqrt{2}}\left(\bar{\sigma}^{I}\right)^{A a} \varphi_{I}, & \varphi^{A a}=\epsilon^{a b} \epsilon^{A B} \varphi_{b B}=\left(\varphi_{b B}\right)^{\dagger},
\end{array}
$$

with $m=1,2,3,4$ and $I=1,2,3,4$. The fermions satisfy the following reality conditions,

$$
\begin{array}{rlrl}
\lambda_{a \alpha} & =-\epsilon_{\alpha \beta} \epsilon_{a b}\left(\lambda_{b \beta}\right)^{\dagger}, & \lambda_{\alpha}^{A}=\epsilon_{\alpha \beta} \epsilon^{A B}\left(\lambda_{\beta}^{B}\right)^{\dagger}, \\
\bar{\lambda}_{a}^{\dot{\alpha}}=-\epsilon^{\dot{\alpha} \dot{\beta}} \epsilon_{a b}\left(\bar{\lambda}_{b}^{\dot{\beta}}\right)^{\dagger}, & \bar{\lambda}^{A \dot{\alpha}}=\epsilon^{\dot{\alpha} \dot{\beta}} \epsilon^{A B}\left(\lambda^{B \dot{\beta}}\right)^{\dagger} .
\end{array}
$$

Open Access. This article is distributed under the terms of the Creative Commons Attribution License (CC-BY 4.0), which permits any use, distribution and reproduction in any medium, provided the original author(s) and source are credited. 


\section{References}

[1] M. Berkooz, M. Rozali and N. Seiberg, Matrix description of M-theory on $T^{4}$ and $T^{5}$, Phys. Lett. B 408 (1997) 105 [hep-th/9704089] [INSPIRE].

[2] N. Seiberg, New theories in six-dimensions and matrix description of M-theory on $T^{5}$ and $T^{5} / Z_{2}$, Phys. Lett. B 408 (1997) 98 [hep-th/9705221] [INSPIRE].

[3] O. Aharony, M. Berkooz, D. Kutasov and N. Seiberg, Linear dilatons, NS five-branes and holography, JHEP 10 (1998) 004 [hep-th/9808149] [INSPIRE].

[4] A. Giveon, D. Kutasov and O. Pelc, Holography for noncritical superstrings, JHEP 10 (1999) 035 [hep-th/9907178] [INSPIRE].

[5] O. Aharony, A brief review of 'little string theories', Class. Quant. Grav. 17 (2000) 929 [hep-th/9911147] [INSPIRE].

[6] E. Witten, On the conformal field theory of the Higgs branch, JHEP 07 (1997) 003 [hep-th/9707093] [INSPIRE].

[7] O. Aharony and M. Berkooz, IR dynamics of $D=2, N=(4,4)$ gauge theories and DLCQ of 'little string theories', JHEP 10 (1999) 030 [hep-th/9909101] [INSPIRE].

[8] A. Giveon and D. Kutasov, Little string theory in a double scaling limit, JHEP 10 (1999) 034 [hep-th/9909110] [INSPIRE].

[9] A. Giveon and D. Kutasov, Comments on double scaled little string theory, JHEP 01 (2000) 023 [hep-th/9911039] [INSPIRE].

[10] C.-M. Chang, Y.-H. Lin, S.-H. Shao, Y. Wang and X. Yin, Little string amplitudes (and the unreasonable effectiveness of 6D SYM), JHEP 12 (2014) 176 [arXiv:1407.7511] [INSPIRE].

[11] Y.-H. Lin, S.-H. Shao, Y. Wang and X. Yin, Interpolating the Coulomb phase of little string theory, JHEP 12 (2015) 022 [arXiv: 1502.01751] [INSPIRE].

[12] N. Seiberg and E. Witten, The D1/D5 system and singular CFT, JHEP 04 (1999) 017 [hep-th/9903224] [INSPIRE].

[13] E. Witten, Branes, instantons, and Taub-NUT spaces, JHEP 06 (2009) 067 [arXiv: 0902.0948] [INSPIRE].

[14] D. Kutasov and D.A. Sahakyan, Comments on the thermodynamics of little string theory, JHEP 02 (2001) 021 [hep-th/0012258] [INSPIRE].

[15] B. Haghighat, A. Iqbal, C. Kozçaz, G. Lockhart and C. Vafa, M-strings, Commun. Math. Phys. 334 (2015) 779 [arXiv:1305.6322] [INSPIRE].

[16] E. Witten, Elliptic genera and quantum field theory, Commun. Math. Phys. 109 (1987) 525 [INSPIRE].

[17] E. Witten, Phases of $N=2$ theories in two-dimensions, Nucl. Phys. B 403 (1993) 159 [hep-th/9301042] [INSPIRE].

[18] A. Gadde and S. Gukov, $2 d$ index and surface operators, JHEP 03 (2014) 080 [arXiv: 1305.0266] [INSPIRE].

[19] F. Benini, R. Eager, K. Hori and Y. Tachikawa, Elliptic genera of two-dimensional $N=2$ gauge theories with rank-one gauge groups, Lett. Math. Phys. 104 (2014) 465 [arXiv:1305.0533] [INSPIRE]. 
[20] F. Benini, R. Eager, K. Hori and Y. Tachikawa, Elliptic genera of $2 d N=2$ gauge theories, Commun. Math. Phys. 333 (2015) 1241 [arXiv:1308.4896] [INSPIRE].

[21] C. Hwang, J. Kim, S. Kim and J. Park, General instanton counting and 5d SCFT, JHEP 07 (2015) 063 [arXiv: 1406.6793] [INSPIRE].

[22] H.-C. Kim, S. Kim, E. Koh, K. Lee and S. Lee, On instantons as Kaluza-Klein modes of M5-branes, JHEP 12 (2011) 031 [arXiv:1110.2175] [INSPIRE].

[23] R. Flume and R. Poghossian, An algorithm for the microscopic evaluation of the coefficients of the Seiberg-Witten prepotential, Int. J. Mod. Phys. A 18 (2003) 2541 [hep-th/0208176] [INSPIRE].

[24] U. Bruzzo, F. Fucito, J.F. Morales and A. Tanzini, Multiinstanton calculus and equivariant cohomology, JHEP 05 (2003) 054 [hep-th/0211108] [INSPIRE].

[25] T.J. Hollowood, A. Iqbal and C. Vafa, Matrix models, geometric engineering and elliptic genera, JHEP 03 (2008) 069 [hep-th/0310272] [INSPIRE].

[26] M.R. Douglas and G.W. Moore, D-branes, quivers and ALE instantons, hep-th/9603167 [INSPIRE].

[27] B. Haghighat, C. Kozcaz, G. Lockhart and C. Vafa, Orbifolds of M-strings, Phys. Rev. D 89 (2014) 046003 [arXiv:1310.1185] [INSPIRE].

[28] D. Tong, The holographic dual of $A d S_{3} \times S^{3} \times S^{3} \times S^{1}$, JHEP 04 (2014) 193 [arXiv: 1402.5135] [INSPIRE].

[29] R. Dijkgraaf, G.W. Moore, E.P. Verlinde and H.L. Verlinde, Elliptic genera of symmetric products and second quantized strings, Commun. Math. Phys. 185 (1997) 197 [hep-th/9608096] [INSPIRE].

[30] E. Witten, Phase transitions in M-theory and F-theory, Nucl. Phys. B 471 (1996) 195 [hep-th/9603150] [INSPIRE].

[31] A. Klemm, P. Mayr and C. Vafa, BPS states of exceptional noncritical strings, in Advanced quantum field theory, La Londe les Maures France (1996), pg. 177 [hep-th/9607139] [INSPIRE].

[32] J. Kim, S. Kim, K. Lee, J. Park and C. Vafa, Elliptic genus of E-strings, arXiv:1411.2324 [INSPIRE].

[33] H.-C. Kim and S. Kim, M5-branes from gauge theories on the 5-sphere, JHEP 05 (2013) 144 [arXiv:1206.6339] [INSPIRE].

[34] G. Lockhart and C. Vafa, Superconformal partition functions and non-perturbative topological strings, arXiv:1210.5909 [INSPIRE].

[35] H.-C. Kim, J. Kim and S. Kim, Instantons on the 5-sphere and M5-branes, arXiv: 1211.0144 [INSPIRE].

[36] H.-C. Kim, S. Kim, S.-S. Kim and K. Lee, The general M5-brane superconformal index, arXiv: 1307.7660 [INSPIRE].

[37] J. Qiu and M. Zabzine, Factorization of $5 D$ super Yang-Mills theory on $Y^{p, q}$ spaces, Phys. Rev. D 89 (2014) 065040 [arXiv:1312.3475] [INSPIRE].

[38] J. Qiu, L. Tizzano, J. Winding and M. Zabzine, Gluing Nekrasov partition functions, Commun. Math. Phys. 337 (2015) 785 [arXiv:1403.2945] [INSPIRE]. 